\title{
Improved non-invasive inverse problem method for the balancing of nonlinear squeeze-film damped rotordynamic systems \\ Dol:
}

10.1016/j.ymssp.2018.07.032

\section{Document Version}

Accepted author manuscript

Link to publication record in Manchester Research Explorer

Citation for published version (APA):

Torres Cedillo, S. G., Al-ghazal, G. G., Bonello, P., \& Cortés Pérez, J. (2019). Improved non-invasive inverse problem method for the balancing of nonlinear squeeze-film damped rotordynamic systems. Mechanical Systems and Signal Processing, 117, 569-593. https://doi.org/10.1016/j.ymssp.2018.07.032

Published in:

Mechanical Systems and Signal Processing

\section{Citing this paper}

Please note that where the full-text provided on Manchester Research Explorer is the Author Accepted Manuscript or Proof version this may differ from the final Published version. If citing, it is advised that you check and use the publisher's definitive version.

\section{General rights}

Copyright and moral rights for the publications made accessible in the Research Explorer are retained by the authors and/or other copyright owners and it is a condition of accessing publications that users recognise and abide by the legal requirements associated with these rights.

\section{Takedown policy}

If you believe that this document breaches copyright please refer to the University of Manchester's Takedown Procedures [http://man.ac.uk/04Y6Bo] or contact uml.scholarlycommunications@manchester.ac.uk providing relevant details, so we can investigate your claim.

\section{OPEN ACCESS}




\title{
Improved non-invasive inverse problem method for the balancing of nonlinear squeeze-film damped rotordynamic systems
}

\author{
Sergio Guillermo Torres Cedillo ${ }^{a}$, Ghaith Ghanim Al-Ghazal ${ }^{b}$, Philip Bonello ${ }^{b}$, Jacinto Cortés Pérez ${ }^{a}$ \\ ${ }^{a}$ Centro Tecnológico Aragón, FES Aragón, Universidad Nacional Autónoma de México (UNAM), Av. \\ Rancho Seco s/n, Edo. de México, México. C.P. 57130. \\ ${ }^{\mathrm{b}}$ School of Mechanical, Aerospace and Civil Engineering, University of Manchester, Manchester UK
}

\begin{abstract}
A non-invasive inverse problem method for rotor balancing relies on casing vibration readings and prior knowledge of the structure. Such a method is important for rotors that are inaccessible under operating conditions. This paper introduces a method for solving the quasi-implicit inverse problem that arises when identifying the required balancing correction for a rotor with only one weak linear connection to the casing, apart from the nonlinear connections. This is typical of aero-engine designs that use a retainer spring with only one of the nonlinear squeeze-film damper (SFD) bearings that support the rotor within the casing. The SFD journal displacements are estimated from casing vibration readings using identified inverse SFD models based on Recurrent Neural Networks (RNNs). The information from these is then used to enhance the condition of the explicit inverse problem set up in previous research for simpler configurations. The methodology is validated using simulated casing vibration readings. The reliability of the RNN inverse SFD models is first demonstrated. The second part of the validation shows that the novel enhanced explicit inverse problem method is essential for effective balancing of this previously unconsidered system. Repeatability and robustness to noise/model uncertainty are satisfactorily demonstrated and limitations discussed.
\end{abstract}

Keywords: Nonlinear vibration; Inverse problem; Rotor balancing; Squeeze-film damper bearings; Recurrent Neural Networks 


\section{Introduction}

Research into unbalance identification and balancing techniques for aircraft engines is increasingly important to the airline industry since efforts to reduce weight result in flexible engine casing structures that are sensitive to unbalance excitation of the rotors [1]. Reliable balancing procedures ensure that passenger comfort, and the structural integrity and lifespan of the aircraft, are not compromised [2].

As illustrated in Figure 1, typical aero-engine assemblies have at least two nested rotors, the Low Pressure (LP) and High Pressure (HP) rotors, that are mounted within a flexible casing via several nonlinear squeeze-film damper (SFD) bearings. The nonlinearity of these bearings comes from the oil film that surrounds the non-rotating outer race of the rolling element bearings. As stated in [3, 1], the LP rotor is relatively easy to balance by traditional methods (discussed below). On the other hand, the HP rotor cannot be accessed under operational conditions because of the restricted space for instrumentation and high temperatures. Hence, in order to monitor a state of unbalance that develops in the HP rotor during its operational lifetime, a non-invasive procedure that is based on vibration measurements at the engine casing, rather than the rotor, is required $[3,1]$ which would then facilitate a "right first time" corrective action.

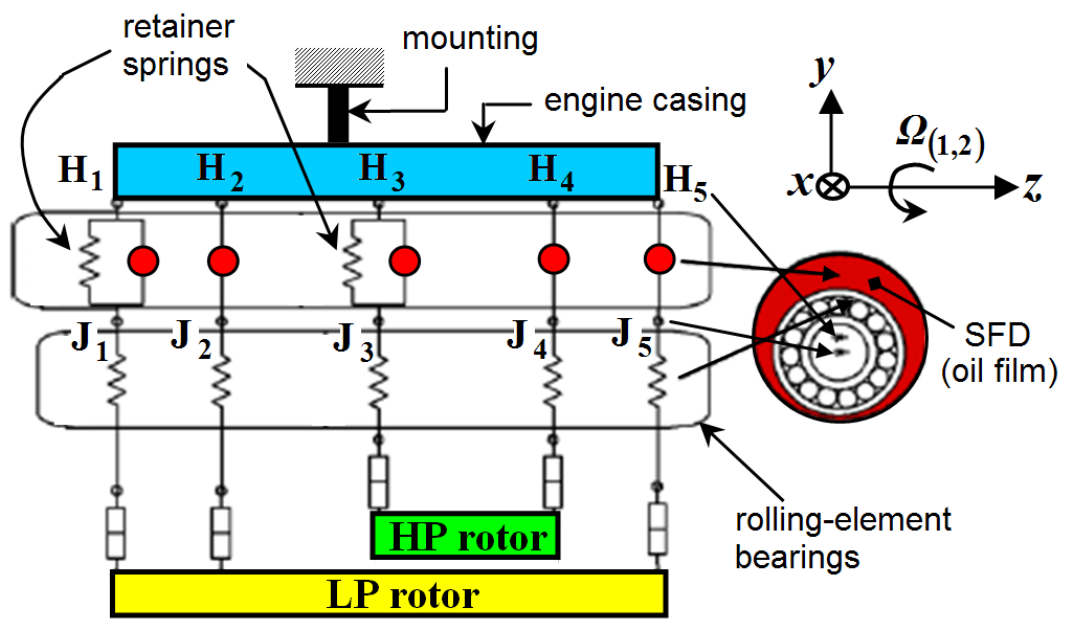

Fig.1. Schematic of a typical twin spool engine [14] 
The identification of the rotor unbalance from vibration measurements at the casing and/or the rotor is referred to as an "inverse" problem [3], in contrast to the "forward" problem, which refers to the prediction of the system vibration in response to a known unbalance distribution. Traditional balancing methods (which include the standard "trim" balancing procedures) [4-8] involve the use of several trial runs and the application of trial masses at fixed balancing planes. Such types of methods are typically based on two representative methods, the influence coefficients balancing method [5] and the modal balancing method [4]. Darlow [6] developed the Unified Balancing Approach (UBA) that combined the advantages of both previous methods. The UBA method involved the calculation of modal trial mass sets, based on the influence coefficient approach of using trial mass data. Foiles et al [7] provided a comprehensive review of the several direct methods for rotor balancing, which were based on the fundamentals of the influence coefficients method and modal method.

Chen et al [9] proposed an optimisation technique based on nonlinear programming to determine the balancing corrections to be applied to prescribed planes. This method required a valid mathematical model of the rotor-dynamic system to use within the optimisation scheme. Unlike the methods of [4-8], the method of Chen et al [9] did not require several trial runs and trial masses since the optimisation was based on measurements from the initial (unbalanced) configuration. However, the method was still invasive since it required measurement of the vibration of the rotor. Moreover, the system model used a linear model for the bearings, rendering it unsuitable for the application of Fig.1. The methods of Krodkiewski et al [10] and Ding et al [11] find the change in unbalance from one rotor condition to another using a known rotordynamic model which can include nonlinear models for the bearings. However, these methods are still invasive since they require that the motion of the rotor journals relative to the bearings can be measured before and after the change in unbalance.

Accordingly, many subsequent efforts have focused on non-invasive inverse problem procedures for identifying the unbalance, which require of prior knowledge of the structure (obtained from modelling or experiment) as in [12-16], [1, 3]. The methods of [12-16] were based on a linear bearing model. In the case of the methods of Lees and Sinha [12-15], although these were applied to a system with journal bearings, they used linearized stiffness and damping coefficients to approximate the nonlinear fluid film forces as linearized functions of the displacements and velocities of the journal relative to the static equilibrium position. This makes such works unsuitable for SFD 
bearing applications like the aero-engine in Fig. 1, where the full nonlinear model is typically considered to be essential for the solution of both forward and inverse problems [1, 3, 17-20].

One of the greatest challenges faced by the analyst attempting to solve the inverse problem for the identification of rotating unbalance using a non-invasive approach is to accommodate bearing nonlinearity, as in the case of SFD bearings used in aero-engines. A review of the literature shows there are very few such works: Dicken et al. [1]; Torres Cedillo and Bonello [3]; Torres Cedillo and Bonello [17]. The first two works, [1] and [3], have considered systems that were simplifications, of varying degrees, of the system in Fig. 1. In both [1] and [3] the LP rotor was omitted from Fig. 1, with focus being placed on the HP rotor and the casing, since it was considered that the LP rotor could be balanced by traditional methods with relatively little effort and cost, as mentioned above. The system considered in the present paper is shown in Fig. 2, which omits the LP rotor as also done in [1] and [3]. As is customary in the analysis of SFD bearings [1, 19], it is assumed that the rolling-element bearing is rigid relative to the associated oil film and/or retainer spring. However, the system in Fig. 2 does not involve the other simplifications made in [1] and [3], which are detailed below.

The work by Dicken et al. [1], started to explore the unbalance identification on rotor-dynamic systems supported by SFDs using vibration measurements from sensors mounted on the engine casing and considering the nonlinearity of the SFD bearing. However, with reference to Fig. 2, the pioneering work in [1] had the following limitations:

1. The analysis considered only one SFD bearing: the SFD on the right hand (RH) side was replaced by a linear spring equal to the stiffness of the rolling-element bearing, leaving only the spring-supported SFD on the left hand (LH) side.

2. The elastic properties of the structure were assumed to be isotropic.

3. Gyroscopic effects were neglected.

4. The whirl vibration of the SFD journal within its housing was assumed to trace a circular centred orbit (CCO) (that has only a synchronous frequency component). 


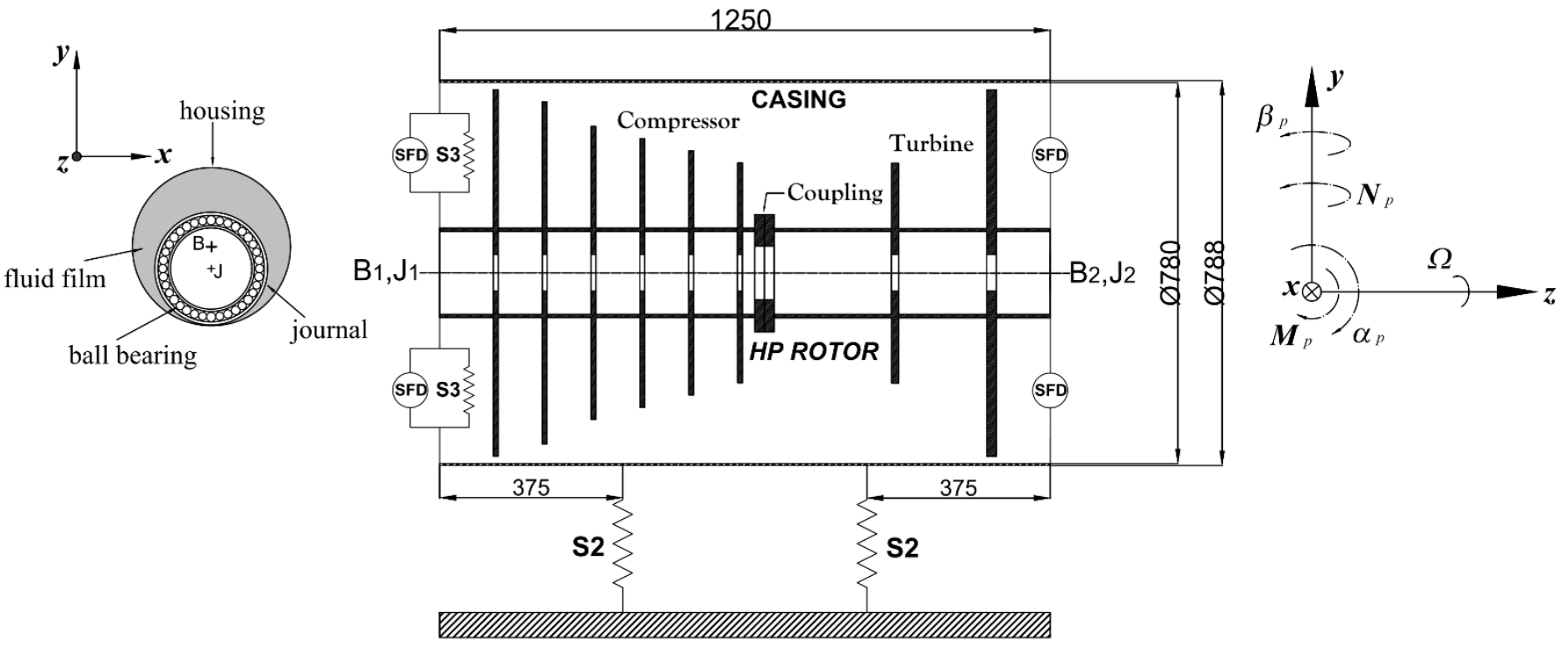

Fig. 2. Flexible rotor/casing system

(dimensions in $\mathrm{mm}$, material and geometric properties of rotor and casing as in [1,3]).

The latter limitation was considered necessary to create a database that related the relative vibration between journal and casing at the SFD to the unbalance, thus enabling an implicit solution of the inverse problem involving nonlinear Tikhonov regularisation [1].

The work by Torres Cedillo and Bonello [3] overcame the above-listed limitations by devising a generic inverse balancing method for SFD rotordynamic systems, which was based on the inversion of the Receptance Harmonic Balance Method (RHBM) $[18,20]$. Hence, the method could be applied for the case of sprung SFDs with a high degree of static eccentricity or unsprung SFDs, provided however that there was at least one strong linear element connecting the source of the unbalance (the rotor) and the measurement location (the casing), apart from the nonlinear connections (SFDs) (which may also be strong). The existence of one linear connection guarantees an explicit relationship between the casing vibration and the unbalance via frequency response functions (FRFs) of the linear part of the structure [3]. This enables the construction of a linear-form operator that could be inverted via a least-squares solution approach. This approach was used in [3] to determine the equivalent unbalance in three prescribed planes from casing vibration data arising from an actual unbalance distribution spread over ten planes. The equivalent unbalance was then used to balance the system and the balancing effectiveness quantified. The method was validated 
on distinct rotor-dynamic systems using simulated casing vibration data. The method was shown to provide effective balancing under hitherto unconsidered practical conditions. The repeatability of the method, as well as its robustness to noise, model uncertainty and balancing errors, were satisfactorily demonstrated. The method in [3] was shown to be effective if there was at least one strong linear connection (between rotor and casing), or at least two relatively weak linear connections. Hence the method in [3] worked well with the following variations of Fig. 2:

- Two linear connections - one weak (low stiffness) and one strong (high stiffness): a sprung SFD on the LH end and rolling element bearing (without SFD) on RH end (as considered by Dicken et al. [1], thus showing that the complicated implicit solution approach used in [1] was not necessary for their system).

- Two linear connections - both weak: a sprung SFD at both ends of the HP rotor.

- One linear connection only, but of high stiffness: a rolling element bearing (without SFD) at one end and an unsprung SFD at the other.

The problem with the method in [3] is that it becomes ineffective for the case of just one weak casing-rotor linear connection, which is the situation in Fig. 2, where the rotor is supported by two SFD bearings, only one of which has a retainer spring. In this case, the explicit inverse problem formulated by FRFs of the linear part of the structure connecting the casing (response measurements) to the rotor (unbalance) becomes badly conditioned since the relationship between casing response and unbalance is quasi-implicit. If there is no linear connection at all (e.g. no retainer springs are used with the SFDs in Fig. 1 or Fig. 2), the relationship is fully implicit (via the SFD forces acting on both casing and rotor, which are a consequence of the unbalance excitation).

In [17], Torres Cedillo and Bonello devised a method for solving a fully implicit nonlinear inverse problem. To solve this problem, it becomes necessary to be able to quantify the shaft vibration at the bearing journal. This was achieved in [17] through identified inverse model of the SFD bearing, which outputs the relative displacement of the journal relative to the bearing housing, for given bearing force inputs. Such an inverse model of the SFD bearing serves as a substitute for internal instrumentation, to enable the solution of the nonlinear inverse problem using external mounted sensors, where is no adequate linear connection between rotor and casing. The nonlinear inverse model of the SFD in [17] was identified from empirical data using a scheme based on a recurrent neural network (RNN). The identified inverse SFD model was then used within the solution of the inverse rotor-bearing problem of a simple non-rotating 
experimental rig with one SFD, for the identification of the rotor excitation force using only accelerometer readings at the flexibly mounted bearing housing.

The novel contribution of the present paper is an effective non-invasive method for balancing generic SFD rotor-dynamic systems that pose a quasi-implicit inverse problem (i.e. with only one weak linear rotor-casing connection, apart from the non-linear connections, as shown in Fig 2). The strategy that is used combines the relative merits of [3] and [17]. The badly-conditioned explicit inverse problem relating the known casing vibration readings with the unknown rotor unbalance is first constructed. An implicit inverse problem relating the unknown vibration of the SFD journals (relative to their bearing housings) with the rotor unbalance is then set up. The relative vibrations at the SFDs in the implicit problem are then determined using identified inverse models for the SFD bearings. These SFD inverse models are based on RNNs and trained to reproduce the Cartesian displacements of the journal relative to the bearing housing, when presented with given input time histories of the Cartesians SFD bearings forces. The partly-solved implicit problem is then combined with the (badly conditioned) explicit inverse problem to construct an enhanced (well-conditioned) explicit inverse problem that can be solved by least squares inversion. The result of the inverse solution is the equivalent unbalance distribution at the prescribed planes of the rotor which is then used to balance the rotor. Finally, the improved method is validated considering different practical scenarios including variations in phase datum and error sources such as noise and model uncertainties. As in [3, 17], the RHBM [18, 20] is employed as underpinning theory. The training data for the RNNs are generated using a "circular chirp excitation" method [21, 22], which is shown by Al-Ghazal et al [22] to be more effective than the method used in [17].

The remaining part of the paper is structured as follows. Section 2 presents the details of the rotordynamic system considered. In section 3, the nonlinear inverse rotordynamic problem is set up. Section 4 describes the identification of the inverse model of the SFD bearings using RNNs. Section 5 presents and discusses the validation test results.

\section{Flexible rotor/casing SFD system}

With reference to Fig. 2, as in [1, 3], the retainer spring S3 is isotropic and has stiffness of $30 \mathrm{MN} / \mathrm{m}$. The present method, as in [3] and unlike [1], can also accommodate the anisotropy of the engine mountings. Therefore, the elastic supports $\mathrm{S} 2$ of the casing are anisotropic in the present case $(300 \mathrm{MN} / \mathrm{m}, 600 \mathrm{MN} / \mathrm{m}$ in horizontal and vertical planes respectively). The sprung SFD on the LH end can accommodate a static offset as in [3], but, unlike [3], the RH SFD 
bearing has no retainer spring. This corresponds to typical engine designs from a leading manufacturer, where only one retainer spring is used per rotor (as shown in Fig. 1) [18].

\subsection{Modal parameters}

As in $[18,20]$ in the 'forward' problem, the inverse problem method presented uses the modes shapes and eigenfrequencies of the linear part of the system under non-rotating conditions, computed from a one-off eigenvalue analysis. Hence, the nonlinear rotating system is regarded as the 'linear part' acted on by the unbalance, static loading and the motion-dependent excitations (nonlinear SFD forces and the gyroscopic effect). The term 'linear part' refers to the system which remains when the SFDs (oil films) in Fig. 2 are replaced by gaps. Hence, the linear part consists of the casing mounted on springs S2, and the rotor, that is supported on the casing via retainer spring S3 at one end and free at the other end. The SFDs are typically assumed to be the only significant source of damping in aero-engine structures [20]. Thus, it is assumed the linear part to be undamped, although the method can easily accommodate proportional damping in the structure of the casing [20]. The finite element (FE) method was used to calculate the mode shapes in each of the horizontal and vertical planes [3]. These mode shapes are determined using a model in NASTRAN [23], where the rotor shaft and cylindrical casing are represented by beam elements and the compressor/turbine disks and couplings are represented by concentrated inertia elements ("disks"). The material and geometric properties are the same as used in [1] and [3]. The first six mode shapes of the linear part of the system in Fig. 2 in the horizontal and vertical planes are shown in Fig. 3(a,b), respectively, where the upper diagram shows the rotor deformation and the lower diagram shows the casing centreline deformation. It is noted that the first mode in each plane will be a $0 \mathrm{~Hz}$ mode defining rigid body pivoting motion of the "sprung-free" rotor about the left-hand sprung support, with no casing motion. 

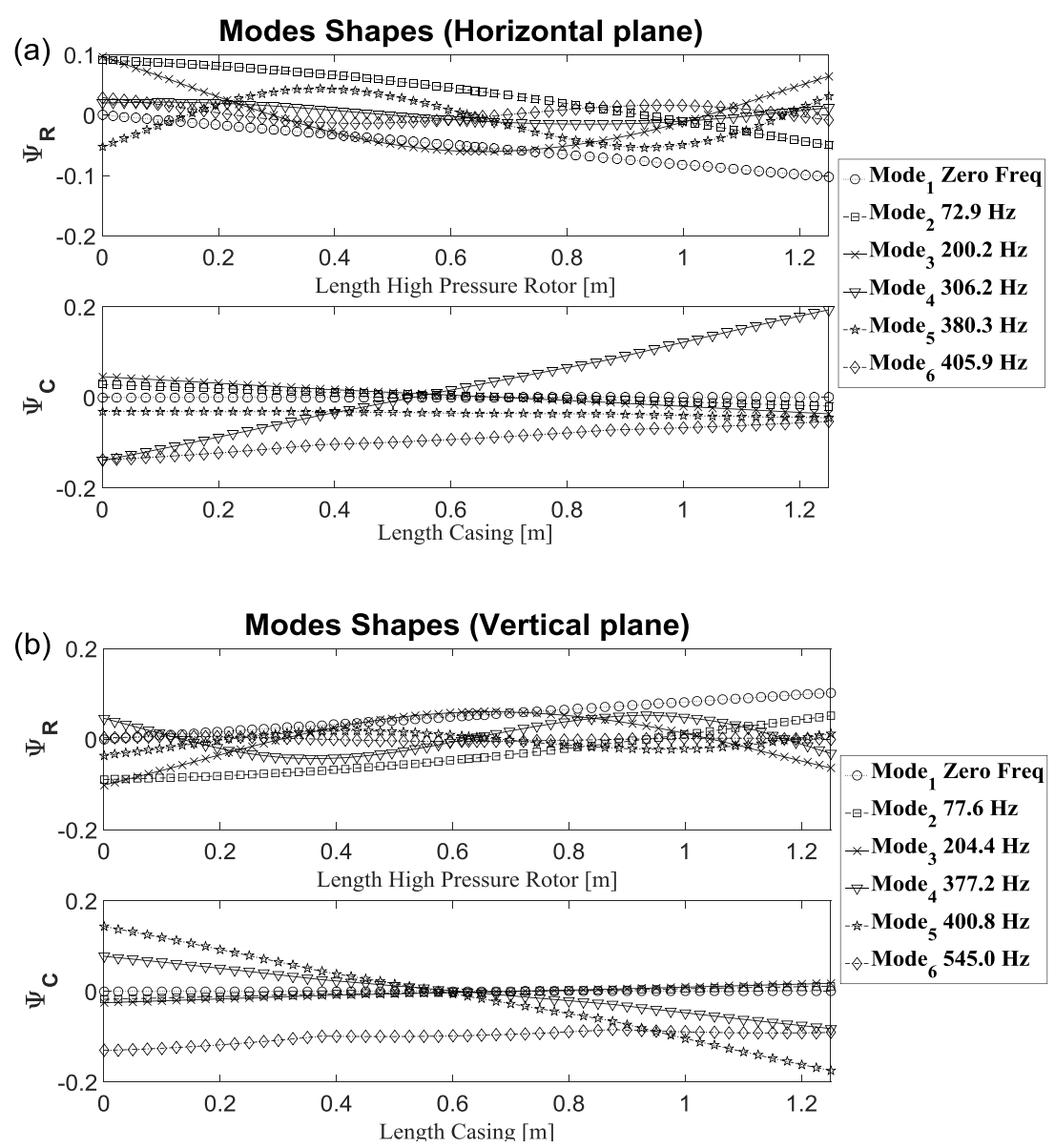

Fig. 3. Eigenforms and Eigenfrequencies for the linear part of the system in Fig. 2:

(a) Horizontal plane. (b) Vertical plane (upper rows refer to rotor deformation, lower rows refer to casing centerline deformation)

\section{Theory}

\subsection{Overview of forward problem}

As in $[3,18,20]$, the equation of motion of the system (Fig. 2 ) in the time domain is transformed into modal space as follows:

$$
\ddot{\mathbf{q}}+\Lambda \mathbf{q}=\sum_{n=1}^{2} \mathbf{H}_{\mathrm{v}_{n}}{ }^{\mathrm{T}} \mathbf{f}_{\mathbf{v}_{n}}\left(\mathbf{v}_{n}, \dot{\mathbf{v}}_{n}\right)+\mathbf{H}_{\mathbf{u}}{ }^{\mathrm{T}} \mathbf{f}_{\mathbf{u}}(t)+\mathbf{H}_{\mathbf{g}}{ }^{\mathrm{T}} \mathbf{g}+\mathbf{H}_{\mathbf{J}_{2}}{ }^{\mathrm{T}} \mathbf{w}
$$

In equation (1), $\mathbf{q}$ is the $R \times 1$ vector of modal coordinates, $\boldsymbol{\Lambda}$ is the diagonal matrix of the squares of the eigenfrequencies $\omega_{1}, \ldots, \omega_{R}$. From the previous section a total of $R=12$ modes is considered, comprising 6 modes in each of the horizontal and vertical planes alternately i.e. with reference to Fig. $3, \omega_{1,2} /(2 \pi)$ respectively correspond 
to $0 \mathrm{~Hz}$ (horizontal plane), $0 \mathrm{~Hz}$ (vertical plane), $\omega_{3,4} /(2 \pi)$ respectively correspond to $72.99 \mathrm{~Hz}$ (horizontal plane), $77.62 \mathrm{~Hz}$ (vertical plane),...etc.

The $2 \times 1$ vector $\mathbf{f}_{\mathbf{v}_{n}}$ comprises the SFD forces in the $x, y$ directions on the journal of SFD no. $n$, which are non-linear functions of the instantaneous displacements and velocities of the journal centre (marked ' $I_{1,2}$ ' in Figure 2 ) relative to the centre of the corresponding housing (marked ' $\mathrm{B}_{1,2}$ ' in Figure 2). The forces on the bearing housings are therefore $-\mathbf{f}_{\mathbf{v}_{n}}$. Let $\mathbf{v}_{n}$ be the vector that contains the instantaneous Cartesians $(x, y)$ displacements of $\mathrm{J}_{n}$ relative to $\mathrm{B}_{n}$. This can be expressed as:

$$
\mathbf{v}_{n}(t)=\left[\begin{array}{c}
x_{\mathrm{rel}, n} \\
y_{\mathrm{rel}, n}
\end{array}\right]=\mathbf{H}_{\mathbf{v}_{n}} \mathbf{q}(t)+\mathbf{e}_{0_{n}}
$$

where the term $\mathbf{H}_{\mathbf{v}_{n}}$ is the $2 \times R$ matrix whose columns are $\boldsymbol{\Psi}_{\mathbf{v}_{n}}^{(1)} \ldots \boldsymbol{\Psi}_{\mathbf{v}_{n}}^{(R)}$

$$
\boldsymbol{\Psi}_{\mathbf{v}_{n}}^{(r)}=\boldsymbol{\Psi}_{\mathbf{J}_{n}}^{(r)}-\boldsymbol{\Psi}_{\mathbf{B}_{n}}^{(r)}, \quad r=1 \ldots R
$$

where $\boldsymbol{\Psi}_{\mathbf{J}_{n}}^{(r)}, \boldsymbol{\Psi}_{\mathbf{B}_{n}}^{(r)}$ are the mass-normalised $2 \times 1$ eigenvectors defining the $x, y$ displacements of the SFD terminals $\mathrm{J}_{n}$, $\mathrm{B}_{n}$ respectively in mode no. $r(r=1 \ldots R)$. In the case of $n=1$, which refers to the LH sprung SFD (Fig. 2), the vector $\mathbf{e}_{0_{n}}$ contains the static offsets of the journal centre $\mathrm{J}_{1}$ relative to the corresponding housing centre $\mathrm{B}_{1}$, in the $x, y$

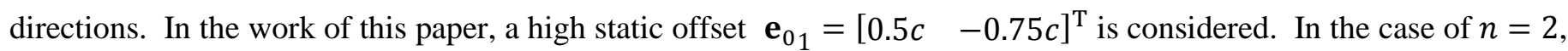
which refers to the RH unsupported SFD, the eccentric mean position is completely determined by the equilibrium between the equivalent rotor weight at the journal and the mean SFD forces $[3,19,20]$. Hence, vector $\mathbf{e}_{02}=\mathbf{0}$ (i.e. it is omitted) and the effect of the static loading on the journal $\mathrm{J}_{2}$ of the RH bearing in the $x, y$ directions is considered in the $2 \times 1$ vector $\mathbf{w}$, which is multiplied by the transpose of matrix $\mathbf{H}_{\mathbf{J}_{2}}$, whose columns are the eigenvectors $\boldsymbol{\Psi}_{\mathbf{J}_{2}}^{(r)}$.

The vector $\mathbf{f}_{\mathbf{u}}$ comprises the unbalance forces, which are defined as follows [3]:

$$
\mathbf{f}_{\mathbf{u}}=\left[\begin{array}{lllllll}
U_{x_{1}} & U_{y_{1}} & U_{x_{2}} & U_{y_{2}} & \cdots & U_{x_{n_{\text {ubb }}}} & U_{y_{n_{\text {ubb }}}}
\end{array}\right]^{\mathrm{T}}
$$

where the unbalance forces $U_{x_{k}}, U_{y_{k}} k=1,2,3, \ldots, n_{\text {unb }}$ are presumed to be concentrated at disk positions $k=$ $1,2,3, \ldots, n_{\mathrm{umb}}:$

$$
U_{x_{k}}=U_{k} \Omega^{2} \sin \left(\Omega t+\gamma+\phi_{k}\right)
$$




$$
U_{y_{k}}=-U_{k} \Omega^{2} \cos \left(\Omega t+\gamma+\phi_{k}\right)
$$

where the unbalance (in kg.m) is denoted by $U_{k}$ at disk no. $k$, and $\phi_{k}$ represents its angular location relative to the arbitrary reference phasor (a marker on the rotor) which is displaced by an angle $\Omega t$ from the (stationary) phase probe, that is at an angle $\gamma$ from the downward vertical (Fig. 4a). Fig. 4b presents the unbalance distribution at ten planes along the HP rotor coinciding with the disks of the system in Fig. 2.

(a)

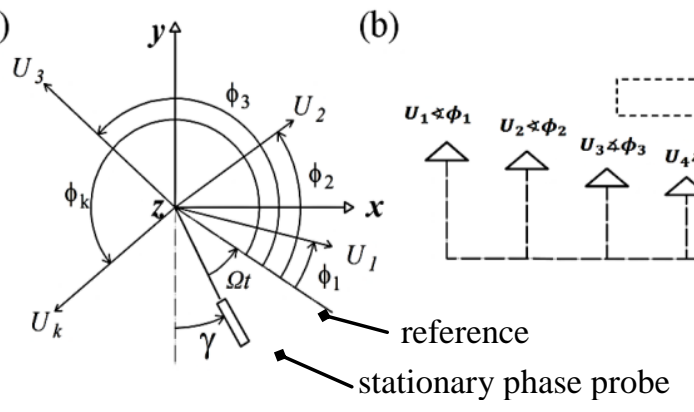

Fig. 4. The distribution of the unbalance forces: (a) angular distribution, with respect to the reference phasor and stationary phase probe; (b) axial distribution over ten positions coinciding with the disks in Fig. 2 [3].

The gyroscopic effect on the rotor is discretised at $G$ positions $[3,18,20]$ and the vector of gyroscopic moments $\mathbf{g}$ is given by:

$$
\mathbf{g}=\mathbf{P}(\Omega) \dot{\boldsymbol{\theta}}
$$

where $\mathbf{g}=\left[\begin{array}{lllll}M_{1} & N_{1} & \ldots & M_{G} & N_{G}\end{array}\right]^{\mathrm{T}}, \boldsymbol{\theta}=\left[\begin{array}{llllll}\beta_{1} & \alpha_{1} & \ldots & \beta_{G} & \alpha_{G}\end{array}\right]^{\mathrm{T}}$ (see the coordinate system of Fig. 2). $\mathbf{P}(\Omega)=$ $\Omega \operatorname{diag}\left\{-I_{1}, I_{1}, \ldots \ldots,-I_{G}, I_{G}\right\}$, where $I_{p}$ is the polar moment of inertia at position $p(p=1, \ldots, G)$. The columns of the modal matrices $\mathbf{H}_{\mathbf{u}}\left(2 n_{\mathrm{unb}} \times R\right)$ and $\mathbf{H}_{\mathbf{g}}(2 G \times R)$ are represented by $\boldsymbol{\Psi}_{\mathbf{u}}^{(r)}, \boldsymbol{\Psi}_{\mathbf{g}}^{(r)}$ respectively and the eigenvectors evaluated at the locations and directions of the entries in $\mathbf{f}_{\mathbf{u}}, \mathbf{g}$.

All data required to solve and validate the inverse problem (which is described in the following section 3.2), including simulated vibration measurements at the casing and input/output data for training the recurrent neural network inverse models of the SFDs (section 4), were produced by solving the forward problem described by eq. (1) (making modifications to the external excitation force term where appropriate). This involved step-by-step integration in the time domain for the modal response $\mathbf{q}(t)$ to a known excitation vector $\mathbf{f}_{\mathbf{u}}(t)$ using the implicit time domain integrator 
ode23s@ available in Matlab [24], as done in [3]. The response time histories at the physical degrees of freedom were then obtained from $\mathbf{q}(t)$ using the appropriate modal transformation matrices [3] e.g. as done in eq. (2).

The SFD bearings were single-land and end-fed with oil of viscosity $0.0049 \mathrm{Nsm}^{-2}$ at a pressure of 3 bar (gauge), as in $[3,18,20]$. The bearing diameter and radial clearance were $220 \mathrm{~mm}, 0.12 \mathrm{~mm}$ respectively, and the land length was 26 mm. During the course of the integration, the pressure distribution in the SFD at each time step was approximated by a combination of the short and long bearing expressions through an "end-leakage" factor of $\lambda=0.10$ which represents the degree of end-sealing, as described in [18]:

$$
p(\vartheta, Z, e, \dot{e}, \dot{\psi})=p_{\text {short }}(\vartheta, Z, e, \dot{e}, \dot{\psi})+\lambda p_{\text {long }}(\vartheta, e, \dot{e}, \dot{\psi})(0.5-Z / L)
$$

$p_{\text {short }}, p_{\text {long }}$ are the short and long bearing approximations respectively [25], $\vartheta, Z$ are spatial coordinates local to the oil film, $L$ the land length and $e, \psi$ are the polar coordinates of the instantaneous position of the journal centre relative to the instantaneous position of the bearing housing centre, that, along with their time derivatives $\dot{e}, \dot{\psi}$, are calculated from $\mathbf{v}_{n}, \dot{\mathbf{v}}_{n}$ via the integrated state variables $\mathbf{q}(t), \dot{\mathbf{q}}(t)$ (eq. (2)). This distribution was truncated below a cavitation pressure of absolute zero $(-101.325 \mathrm{kPa})$ and numerically integrated across the oil film to generate the instantaneous SFD forces $\mathbf{f}_{\mathbf{v}_{n}}(t)$ [19].

\subsection{Construction of the inverse problem}

It is assumed that the vibration is periodic at a fundamental frequency equal to the rotational speed $\Omega$, in which case the $2 \times 1$ vector of the vibration velocity readings in the $x, y$ directions at each casing location $P_{i}, i=1 \ldots n_{\mathrm{s}}$, can be expressed as [3]:

$$
\mathbf{z}_{\mathbf{p}_{i}}=\sum_{s=1}^{m} \mathbf{z}_{\mathbf{p}_{i_{\mathrm{c}}}}^{(s)} \cos (s \Omega t)+\mathbf{z}_{\mathbf{p}_{i_{s}}}^{(s)} \sin (s \Omega t)
$$

where

$$
\mathbf{z}_{\mathbf{p}_{i}}=\dot{\mathbf{x}}_{\mathbf{p}_{i}}, \quad \mathbf{x}_{\mathbf{p}_{i}}=\left[\begin{array}{l}
x_{\mathbf{p}_{i}}(t) \\
y_{\mathbf{p}_{i}}(t)
\end{array}\right]
$$

and 


$$
\mathbf{x}_{\mathbf{p}_{i}}=\overline{\mathbf{x}}_{\mathbf{p}_{i}}+\sum_{s=1}^{m} \mathbf{x}_{\mathbf{p}_{i_{\mathrm{c}}}}^{(s)} \cos (s \Omega t)+\mathbf{x}_{\mathbf{p}_{i_{\mathrm{s}}}}^{(s)} \sin (s \Omega t)
$$

With reference to eq. (1), defining

$$
\mathbf{f}_{\mathbf{v}}=\left[\begin{array}{l}
\mathbf{f}_{\mathbf{v}_{1}}\left(\mathbf{v}_{1}, \dot{\mathbf{v}}_{1}\right) \\
\mathbf{f}_{\mathbf{v}_{2}}\left(\mathbf{v}_{2}, \dot{\mathbf{v}}_{2}\right)
\end{array}\right], \mathbf{v}=\left[\begin{array}{l}
\mathbf{v}_{1} \\
\mathbf{v}_{2}
\end{array}\right]
$$

the $4 \times 1$ vectors $\mathbf{f}_{\mathbf{v}}, \mathbf{v}$ can therefore also be expressed as:

$$
\begin{aligned}
& \mathbf{f}_{\mathbf{v}}=\overline{\mathbf{f}}_{\mathbf{v}}+\sum_{s=1}^{m} \mathbf{f}_{\mathbf{v}_{\mathbf{c}}}^{(s)} \cos (s \Omega t)+\mathbf{f}_{\mathbf{v}_{\mathbf{S}}}^{(s)} \sin (s \Omega t) \\
& \mathbf{v}=\overline{\mathbf{v}}+\sum_{s=1}^{m} \mathbf{v}_{\mathrm{C}}^{(s)} \cos (s \Omega t)+\mathbf{v}_{\mathrm{S}}^{(s)} \sin (s \Omega t)
\end{aligned}
$$

The casing vibration readings $\mathbf{z}_{\mathbf{p}_{i}}$ are the result of the actual unbalance distribution shown in Fig. 4, which is described by the actual excitation vector $\mathbf{f}_{\mathbf{u}}$ (eq. (4)). The inverse problem seeks to identify an equivalent unbalance force vector $\mathbf{f}_{\widehat{\mathbf{u}}}$ that acts in a prescribed set of planes where the balancing masses can then be applied. In this work, three balancing planes are used, as shown in Fig. 5. Of course, the actual unbalances $U_{1}, U_{2}, U_{3}, \ldots$ will generally reside in more than three planes (Figure 4(b)) and the three equivalent unbalances $\widehat{U}_{1}, \widehat{U}_{2}, \widehat{U}_{3}$ will not be at the locations of $U_{1}, U_{2}, U_{3}$ (Fig. 5). However, the angular displacements $\hat{\phi}_{k}$ are with respect to the same reference phasor marker (the line that is displaced by $\Omega t$ from the phase probe in Fig. 4(a) and Fig. 5(a)). It is noted that the locations of $\widehat{U}_{1}, \widehat{U}_{2}, \widehat{U}_{3}$ are the balancing planes, where unbalances equal and opposite to $\widehat{U}_{1}, \widehat{U}_{2}, \widehat{U}_{3}$ will be applied once these are estimated. 
(a)

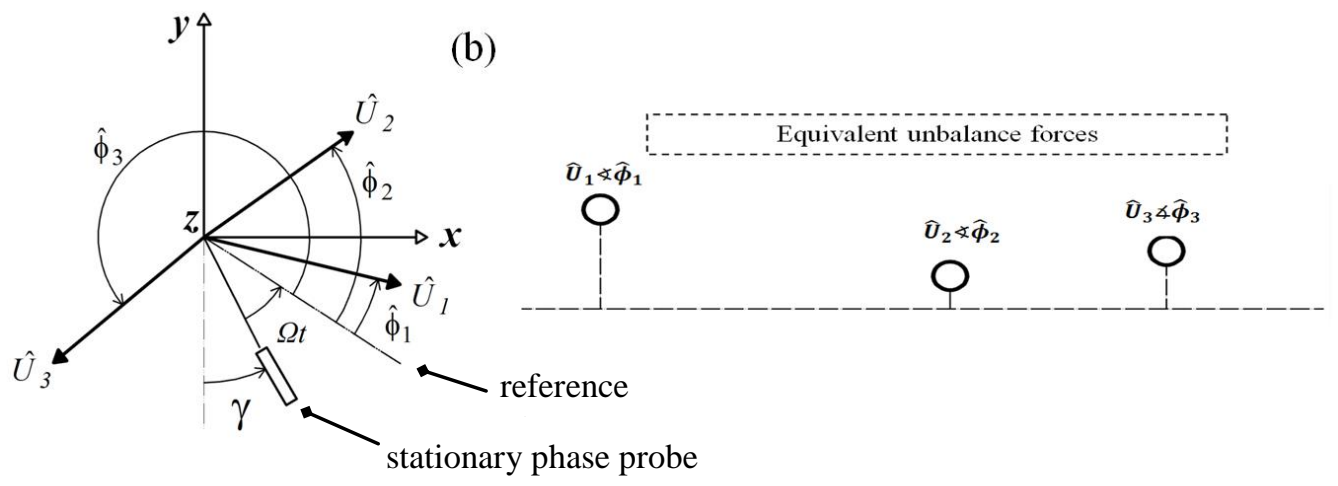

Fig. 5. Equivalent unbalance forces: (a) angular distribution of unbalance, with respect to reference phasor and stationary phase probe; (b) balancing planes.

The equivalent unbalance force vector is given by:

$$
\mathbf{f}_{\widehat{\mathbf{u}}}=\Omega^{2}\left\{\widehat{\mathbf{u}}_{\mathrm{C}} \cos (\Omega t)+\widehat{\mathbf{u}}_{\mathrm{S}} \sin (\Omega t)\right\}
$$

where the elements of $\widehat{\mathbf{u}}_{\mathrm{C}}, \widehat{\mathbf{u}}_{\mathrm{S}}$ are the ultimate unknowns of the inverse problem. With reference to the penultimate paragraph of the Introduction, the following section (3.2.1) shall construct the explicit inverse problem, which is badly conditioned for the system considered (Fig. 2). Section 3.2.2 shall present the implicit problem and its partial solution for the vibration of the SFD journals relative to their housings using RNNs. Section 3.2.3 shall present the final expression for the enhanced inverse problem, which combines the explicit problem with the partly-solved implicit problem.

\subsubsection{Explicit inverse problem}

Using the same nomenclature as in [3], one can use RHBM to relate the synchronous Fourier coefficients of the measured vibration velocity readings at each casing location with the Fourier coefficients of the equivalent unbalance force vector (that acts in a prescribed set of planes where the balancing masses are then applied): 


$$
\left[\begin{array}{c}
\mathbf{z}_{\mathbf{p}_{i \mathrm{~S}}}^{(1)} \\
\mathbf{z}_{\mathbf{p}_{i \mathrm{C}}}^{(1)}
\end{array}\right]=\mathbf{A}_{i}(\Omega)\left[\begin{array}{c}
\mathbf{f}_{\mathbf{v}_{\mathrm{c}}}^{(1)} \\
\mathbf{f}_{\mathbf{v}_{\mathrm{S}}}^{(1)}
\end{array}\right]+\mathbf{B}_{i}(\Omega)\left[\begin{array}{c}
\widehat{\mathbf{u}}_{\mathrm{C}} \\
\widehat{\mathbf{u}}_{\mathrm{S}}
\end{array}\right] \quad\left(i=1 \ldots n_{\mathrm{S}}\right)
$$

where

$$
\begin{gathered}
\mathbf{A}_{i}(\Omega)=\left[\begin{array}{cc}
-\mathbf{M}_{\mathbf{p}_{i} \mathbf{v}}(\Omega)+\mathbf{E}_{i}(\Omega) & -\mathbf{F}_{i}(\Omega) \\
-\mathbf{F}_{i}(\Omega) & \mathbf{M}_{\mathbf{p}_{i} \mathbf{v}}(\Omega)-\mathbf{E}_{i}(\Omega)
\end{array}\right] \\
\mathbf{B}_{i}(\Omega)=\Omega^{2}\left[\begin{array}{cc}
-\mathbf{M}_{\mathbf{p}_{i} \widehat{\mathbf{u}}}(\Omega)+\mathbf{L}_{i}(\Omega) & -\mathbf{Q}_{i}(\Omega) \\
-\mathbf{Q}_{i}(\Omega) & \mathbf{M}_{\mathbf{p}_{i} \widehat{\mathbf{u}}}(\Omega)-\mathbf{L}_{i}(\Omega)
\end{array}\right] \\
\mathbf{M}_{\mathbf{p}_{i} \mathbf{v}}=\Omega \mathbf{C}_{\mathbf{p}_{i} \mathbf{v}}(\Omega), \mathbf{M}_{\mathbf{p}_{i} \mathbf{g}}(\Omega)=\Omega \mathbf{C}_{\mathbf{p}_{i} \mathbf{g}}(\Omega), \\
\mathbf{E}_{i}(\Omega)=\Omega \mathbf{M}_{\mathbf{p}_{i} \widehat{\mathbf{u}}}=\Omega \mathbf{C}_{\mathbf{p}_{i} \widehat{\mathbf{u}}}(\Omega) \\
\mathbf{F}_{i}(\Omega)=\Omega \mathbf{M}_{\mathbf{p}_{i} \mathbf{g}}(\Omega) \mathbf{P}(\Omega) \mathbf{P}(\Omega) \mathbf{D}(\Omega) \mathbf{C}_{\theta \mathbf{v}}(\Omega) \\
\mathbf{L}_{i}(\Omega)=\Omega \mathbf{M}_{\mathbf{p}_{i} \mathbf{g}}(\Omega) \mathbf{P}(\Omega) \mathbf{D}(\Omega) \breve{\mathbf{B}}(\Omega) \mathbf{C}_{\theta \widehat{\mathbf{u}}}(\Omega) \\
\mathbf{Q}_{i}(\Omega)=\Omega \mathbf{M}_{\mathbf{p}_{i} \mathbf{g}}(\Omega) \mathbf{P}(\Omega) \mathbf{D}(\Omega) \mathbf{C}_{\theta \widehat{\mathbf{u}}}(\Omega) \\
\mathbf{D}(\Omega)=\left(\mathbf{I}+(\breve{\mathbf{B}})^{2}\right) \\
\breve{\mathbf{B}}(\Omega)=\Omega \mathbf{C}_{\theta \mathbf{g}}(\Omega) \mathbf{P}(\Omega)
\end{gathered}
$$

where the diagonal matrix $\mathbf{P}(\Omega)$ was previously defined under eq. (6). In the above equations, the matrices $\mathbf{C}_{\mathbf{p}_{i} \mathbf{v}}$, $\mathbf{C}_{\mathbf{p}_{i} \mathbf{g}}, \ldots$ etc are FRF (receptance) matrices defined from modal theory [26], [18] as:

$$
\begin{gathered}
\mathbf{C}_{\mathbf{p}_{i} \mathbf{v}}(\Omega)=\sum_{r=1}^{R} \frac{\boldsymbol{\psi}_{\mathbf{p}_{i}}^{(r)} \boldsymbol{\psi}_{\mathbf{v}}^{(r)^{\mathrm{T}}}}{\omega_{r}^{2}-\Omega^{2}} \\
\mathbf{C}_{\mathbf{p}_{i} \mathbf{g}}(\Omega)=\sum_{r=1}^{R} \frac{\left.\boldsymbol{\Psi}_{\mathbf{p}_{i}}^{(r)} \boldsymbol{\Psi}_{\mathbf{g}}^{(r)}\right)^{\mathrm{T}}}{\omega_{r}^{2}-\Omega^{2}} \\
\mathbf{C}_{\mathbf{p}_{i} \hat{\mathbf{u}}}(\Omega)=\sum_{r=1}^{R} \frac{\left.\boldsymbol{\Psi}_{\mathbf{p}_{i}}^{(r)} \boldsymbol{\Psi}_{\mathbf{u}}^{(r)}\right)^{\mathrm{T}}}{\omega_{r}^{2}-\Omega^{2}} \\
\mathbf{C}_{\boldsymbol{\theta} \mathbf{v}}(\Omega)=\sum_{r=1}^{R} \frac{\left.\boldsymbol{\Psi}_{\theta}^{(r)} \boldsymbol{\Psi}_{\mathbf{v}}^{(r)}\right)^{\mathrm{T}}}{\omega_{r}^{2}-\Omega^{2}}
\end{gathered}
$$




$$
\begin{aligned}
& \mathbf{C}_{\boldsymbol{\theta g}}(\Omega)=\sum_{r=1}^{R} \frac{\boldsymbol{\Psi}_{\boldsymbol{\theta}}^{(r)} \boldsymbol{\Psi}_{\mathbf{g}}^{(r)^{\mathrm{T}}}}{\omega_{r}^{2}-\Omega^{2}} \\
& \mathbf{C}_{\boldsymbol{\theta} \widehat{\mathbf{u}}}(\Omega)=\sum_{r=1}^{R} \frac{\boldsymbol{\Psi}_{\boldsymbol{\theta}}^{(r)} \boldsymbol{\Psi}_{\widehat{\mathbf{u}}}^{(r)^{\mathrm{T}}}}{\omega_{r}^{2}-\Omega^{2}}
\end{aligned}
$$

where, with reference to eq. (3):

$$
\boldsymbol{\Psi}_{\mathbf{v}}^{(r)}=\left[\begin{array}{c}
\boldsymbol{\Psi}_{\mathbf{v}_{1}}^{(r)} \\
\boldsymbol{\Psi}_{\mathbf{v}_{2}}^{(r)}
\end{array}\right]
$$

and $\boldsymbol{\Psi}_{\mathbf{p}_{i}}^{(r)}, \boldsymbol{\Psi}_{\widehat{\mathbf{u}}}^{(r)}, \boldsymbol{\Psi}_{\mathrm{g}}^{(r)}, \boldsymbol{\Psi}_{\boldsymbol{\theta}}^{(r)}$ are the eigenvectors evaluated at the degrees of freedom corresponding to the entries in $\mathbf{x}_{\mathbf{p}_{i}}, \mathbf{f}_{\widehat{\mathbf{u}}}, \mathbf{g}$, and $\boldsymbol{\theta}$ respectively in mode no. $r(r=1 \ldots R)$.

Previous work [3] demonstrated that the $8 \times 1$ vector of synchronous Fourier coefficients $\left[\begin{array}{l}\mathbf{f}_{\mathbf{v}_{\mathbf{c}}}^{(1)} \\ \mathbf{f}_{\mathbf{v}_{\mathbf{s}}}^{(1)}\end{array}\right]$ at the two SFD locations appearing in eq. (17) can be expressed in terms of the unbalance and the synchronous components of the velocity measurements at an equal number of reference sensor locations. As in [3], the current case-study employs 6 sensors locations, which are evenly distributed along the casing (see Figure 6.). $\mathrm{P}_{1}$ and $\mathrm{P}_{6}$ are chosen as the reference locations since these conventiently coincide with the axial locations of the SFDs.

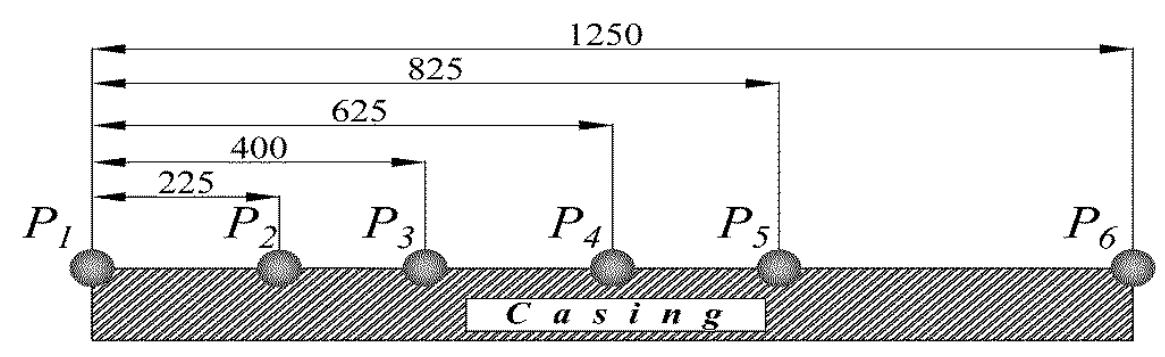

Fig. 6. The locations of the sensor over the casing (dimensions in $\mathrm{mm}$ ). 
Referring eq. (17) to points $P_{1}$ and $P_{6}$ and rearranging:

$$
\left[\begin{array}{c}
\mathbf{f}_{\mathbf{v}_{\mathrm{C}}}^{(1)} \\
\mathbf{f}_{\mathbf{v}_{\mathrm{S}}}^{(1)}
\end{array}\right]=\left[\begin{array}{l}
\mathbf{A}_{\mathbf{1}}(\Omega) \\
\mathbf{A}_{\mathbf{6}}(\Omega)
\end{array}\right]^{-\mathbf{1}}\left[\begin{array}{c}
\mathbf{z}_{\mathbf{p}_{1 \mathrm{~S}}}^{(1)} \\
\mathbf{z}_{\mathbf{p}_{\mathbf{1}}}^{(1)} \\
\mathbf{z}_{\mathbf{p}_{\mathbf{6}_{\mathrm{S}}}^{(1)}}^{(1)} \\
\mathbf{z}_{\mathbf{p}_{\mathbf{6}}}^{(1)}
\end{array}\right]-\left[\begin{array}{l}
\mathbf{A}_{\mathbf{1}}(\Omega) \\
\mathbf{A}_{\mathbf{6}}(\Omega)
\end{array}\right]^{-\mathbf{1}}\left[\begin{array}{l}
\mathbf{B}_{\mathbf{1}}(\Omega) \\
\mathbf{B}_{\mathbf{6}}(\Omega)
\end{array}\right]\left[\begin{array}{l}
\widehat{\mathbf{u}}_{\mathrm{C}} \\
\widehat{\mathbf{u}}_{\mathrm{S}}
\end{array}\right]
$$

The above expression for the vector $\left[\begin{array}{l}\mathbf{f}_{\mathbf{v}_{\mathbf{c}}}^{(1)} \\ \mathbf{f}_{\mathbf{v}_{\mathbf{s}}}^{(1)}\end{array}\right]$ can be back-substituted into eq. (17) for the remaining values of $i$ (i.e. for $i=2,3,4$, and the resulting equations expressed as:

$$
\mathbf{z}(\Omega)=\mathbf{U}(\Omega)\left[\begin{array}{l}
\widehat{\mathbf{u}}_{\mathrm{C}} \\
\widehat{\mathbf{u}}_{\mathrm{S}}
\end{array}\right]
$$

where:

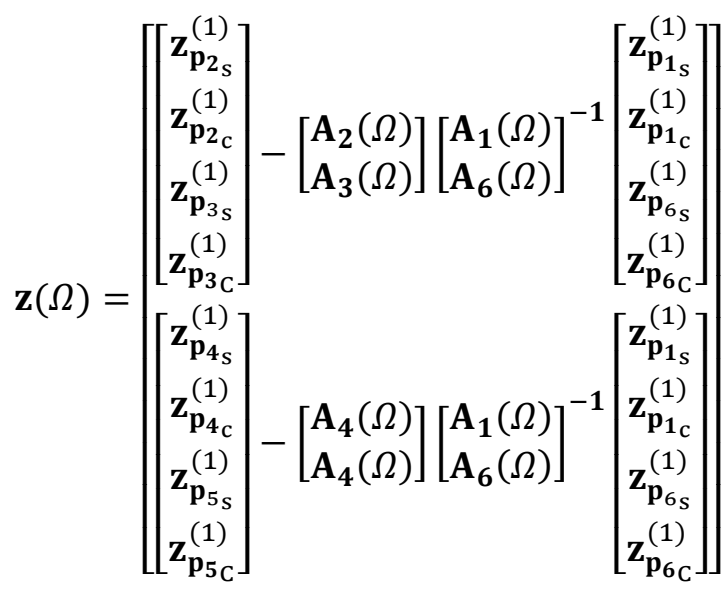

and

$$
\mathbf{U}(\Omega)=\left[\begin{array}{l}
{\left[\begin{array}{l}
\mathbf{B}_{2}(\Omega) \\
\mathbf{B}_{3}(\Omega)
\end{array}\right]-\left[\begin{array}{l}
\mathbf{A}_{2}(\Omega) \\
\mathbf{A}_{3}(\Omega)
\end{array}\right]\left[\begin{array}{l}
\mathbf{A}_{1}(\Omega) \\
\mathbf{A}_{\mathbf{6}}(\Omega)
\end{array}\right]^{-\mathbf{1}}\left[\begin{array}{l}
\mathbf{B}_{\mathbf{1}}(\Omega) \\
\mathbf{B}_{\mathbf{6}}(\Omega)
\end{array}\right]} \\
{\left[\begin{array}{l}
\mathbf{B}_{\mathbf{4}}(\Omega) \\
\mathbf{B}_{\mathbf{5}}(\Omega)
\end{array}\right]-\left[\begin{array}{l}
\mathbf{A}_{4}(\Omega) \\
\mathbf{A}_{\mathbf{5}}(\Omega)
\end{array}\right]\left[\begin{array}{l}
\mathbf{A}_{1}(\Omega) \\
\mathbf{A}_{\mathbf{6}}(\Omega)
\end{array}\right]^{-\mathbf{1}}\left[\begin{array}{l}
\mathbf{B}_{\mathbf{1}}(\Omega) \\
\mathbf{B}_{\mathbf{6}}(\Omega)
\end{array}\right]}
\end{array}\right]
$$


Eq. (30) is referred in the present study as the explicit inverse problem at one operational speed $\Omega$, since it can be solved for the unbalance terms by least squares inversion when augmented over several speeds. However, as stated in the Introduction, for the type of system of this paper (Fig. 2), this problem is badly conditioned.

\subsubsection{Implicit inverse problem}

In a manner analogous to eq. (17), one can use $\operatorname{RHBM}[18,20]$ to relate the synchronous Fourier coefficients of the relative displacements at the SFDs (eq. (15)) with the Fourier coefficients of the equivalent unbalance force vector:

$$
\left[\begin{array}{c}
\mathbf{v}_{\mathrm{C}}^{(1)} \\
\mathbf{v}_{\mathrm{S}}^{(1)}
\end{array}\right]=\widetilde{\mathbf{A}}(\Omega)\left[\begin{array}{c}
\mathbf{f}_{\mathbf{v}_{\mathrm{c}}}^{(1)} \\
\mathbf{f}_{\mathbf{v}_{\mathrm{S}}}^{(1)}
\end{array}\right]+\widetilde{\mathbf{U}}(\Omega)\left[\begin{array}{c}
\widehat{\mathbf{u}}_{\mathrm{C}} \\
\widehat{\mathbf{u}}_{\mathrm{S}}
\end{array}\right]
$$

where:

$$
\begin{gathered}
\widetilde{\mathbf{A}}(\Omega)=\left[\begin{array}{cc}
\mathbf{C}_{\mathbf{v v}}(\Omega)-\tilde{\mathbf{E}}(\Omega) & \tilde{\mathbf{F}}(\Omega) \\
-\tilde{\mathbf{F}}(\Omega) & \mathbf{C}_{\mathbf{v v}}(\Omega)-\tilde{\mathbf{E}}(\Omega)
\end{array}\right] \\
\widetilde{\mathbf{U}}(\Omega)=\Omega^{2}\left[\begin{array}{cc}
\mathbf{C}_{\mathbf{v} \widehat{\mathbf{u}}}(\Omega)-\tilde{\mathbf{L}}(\Omega) & \widetilde{\mathbf{Q}}(\Omega) \\
-\widetilde{\mathbf{Q}}(\Omega) & \mathbf{C}_{\mathbf{v} \widehat{\mathbf{u}}}(\Omega)-\tilde{\mathbf{L}}(\Omega)
\end{array}\right]
\end{gathered}
$$

where

$$
\begin{aligned}
\tilde{\mathbf{E}}(\Omega) & =\Omega \mathbf{C}_{\mathbf{v g}}(\Omega) \mathbf{P}(\Omega) \mathbf{D}(\Omega) \breve{\mathbf{B}}(\Omega) \mathbf{C}_{\theta \mathbf{v}}(\Omega) \\
\tilde{\mathbf{F}}(\Omega) & =\Omega \mathbf{C}_{\mathbf{v g}}(\Omega) \mathbf{P}(\Omega) \mathbf{D}(\Omega) \mathbf{C}_{\theta \mathbf{v}}(\Omega) \\
\tilde{\mathbf{L}}(\Omega) & =\Omega \mathbf{C}_{\mathbf{v g}}(\Omega) \mathbf{P}(\Omega) \mathbf{D}(\Omega) \widetilde{\mathbf{B}}(\Omega) \mathbf{C}_{\theta \widehat{\mathbf{u}}}(\Omega) \\
\widetilde{\mathbf{Q}}(\Omega) & =\Omega \mathbf{C}_{\mathbf{v g}}(\Omega) \mathbf{P}(\Omega) \mathbf{D}(\Omega) \mathbf{C}_{\theta \widehat{\mathbf{u}}}(\Omega)
\end{aligned}
$$

The matrix $\mathbf{D}(\Omega)$ was previously defined in eq. (25). Similarly as the previous sub-section, the receptance matrices are defined, as: 


$$
\begin{aligned}
& \mathbf{C}_{\mathbf{v v}}(\Omega)=\sum_{r=1}^{R} \frac{\boldsymbol{\Psi}_{\mathbf{v}}^{(r)} \boldsymbol{\Psi}_{\mathbf{v}}^{(r)^{T}}}{\omega_{r}^{2}-\Omega^{2}} \\
& \mathbf{C}_{\mathbf{v g}}(\Omega)=\sum_{r=1}^{R} \frac{\boldsymbol{\Psi}_{\mathbf{v}}^{(r)} \boldsymbol{\Psi}_{\mathbf{g}}^{(r)^{T}}}{\omega_{r}^{2}-\Omega^{2}} \\
& \mathbf{C}_{\mathbf{v} \widehat{\mathbf{u}}}(\Omega)=\sum_{r=1}^{R} \frac{\boldsymbol{\Psi}_{\mathbf{v}}^{(r)} \boldsymbol{\Psi}_{\widehat{\mathbf{u}}}^{(r)}}{\omega_{r}^{2}-\Omega^{2}}
\end{aligned}
$$

The remaining receptance matrices $\mathbf{C}_{\theta \mathbf{v}}(\Omega)$ and $\mathbf{C}_{\theta \widehat{\mathbf{u}}}(\Omega)$ were also previously defined in Eqs. (27d) and (27f). Eq. (33) can be rearranged to obtain the following equation:

$$
\tilde{\mathbf{z}}(\Omega)=\widetilde{\mathbf{U}}(\Omega)\left[\begin{array}{l}
\widehat{\mathbf{u}}_{\mathrm{C}} \\
\widehat{\mathbf{u}}_{\mathrm{S}}
\end{array}\right]
$$

where:

$$
\tilde{\mathbf{z}}(\Omega)=\left[\begin{array}{l}
\mathbf{v}_{\mathrm{C}}^{(1)} \\
\mathbf{v}_{\mathbf{S}}^{(1)}
\end{array}\right]-\widetilde{\mathbf{A}}(\Omega)\left[\begin{array}{c}
\mathbf{f}_{\mathbf{v}_{\mathrm{c}}}^{(1)} \\
\mathbf{f}_{\mathbf{v}_{\mathbf{S}}}^{(1)}
\end{array}\right]
$$

Eq. (41) is referred in the present study as the implicit inverse problem at one operational speed $\Omega$, since, in contrast to eq. (30), the left hand term $\tilde{\mathbf{z}}(\Omega)$ contains the Fourier coefficients of the SFD journals relative to the housing, which cannot be determined from sensors attached to the casing. However, as proposed in this paper, an approximation for $\tilde{\mathbf{z}}(\Omega)$, denoted by $\left.\tilde{\mathbf{z}}(\Omega)\right|_{\mathrm{a}}$, can be obtained as follows:

a) neglect the weak linear connection between the rotor and casing (i.e. the retainer spring in Fig. 2);

b) use identified inverse models of the SFD bearings, based on recurrent neural networks (RNNs), which output the relative displacement time history for known bearing force time history.

Step (a) enables the approximate determination of the periodic time history of the SFD forces from the vibration velocity readings of the sensors on the engine casing. By neglecting the weak linear connection between rotor and casing, the receptance FRFs relating excitations on the rotor (unbalance, gyroscopic moments) with the response at the casing are assumed to be negligible. Hence, using RHBM, each harmonic of the vibration response readings at the two reference locations $\mathrm{P}_{1}$ and $\mathrm{P}_{6}$ (Fig. 6) can be expressed in terms of the corresponding harmonic of the SFD forces $-\mathbf{f}_{\mathbf{v}}$ acting on the casing at the bearing housings as follows: 


$$
\left[\begin{array}{c}
\mathbf{z}_{\mathbf{p}_{1 \mathrm{~S}}}^{(s)} \\
\mathbf{z}_{\mathbf{p}_{\mathbf{1}_{\mathrm{C}}}}^{(s)} \\
\mathbf{z}_{\mathbf{p}_{\mathbf{p}_{\mathrm{S}}}}^{(s)} \\
\mathbf{z}_{\mathbf{p}_{\mathbf{6}}}^{(s)}
\end{array}\right] \approx\left[\begin{array}{cc}
-\mathbf{M}_{\mathbf{p}_{\mathbf{1}} \mathbf{B}}(s \Omega) & \mathbf{0} \\
\mathbf{0} & \mathbf{M}_{\mathbf{p}_{\mathbf{1}} \mathbf{B}}(s \Omega) \\
-\mathbf{M}_{\mathbf{p}_{\mathbf{2}} \mathbf{B}}(s \Omega) & \mathbf{0} \\
\mathbf{0} & \mathbf{M}_{\mathbf{p}_{2} \mathbf{B}}(s \Omega)
\end{array}\right]\left[\begin{array}{c}
-\mathbf{f}_{\mathbf{v}_{\mathrm{c}}}^{(s)} \\
-\mathbf{f}_{\mathbf{v}_{\mathrm{S}}}^{(s)}
\end{array}\right], \quad s=1 \ldots \ldots m .
$$

where

$$
\mathbf{M}_{\mathbf{p}_{i} \mathbf{B}}=s \Omega \mathbf{C}_{\mathbf{p}_{i} \mathbf{B}}(s \Omega), \quad \mathbf{C}_{\mathbf{p}_{i} \mathbf{B}}(s \Omega)=\sum_{r=1}^{R} \frac{\boldsymbol{\Psi}_{\mathbf{P}_{i}}^{(r)} \boldsymbol{\psi}_{\mathbf{B}}^{(r)}}{\omega_{r}^{2}-(s \Omega)^{2}}
$$

and, with reference to eq. (45):

$$
\boldsymbol{\Psi}_{\mathrm{B}}^{(r)}=\left[\begin{array}{l}
\boldsymbol{\Psi}_{\mathrm{B}_{1}}^{(r)} \\
\boldsymbol{\Psi}_{\mathrm{B}_{2}}^{(r)}
\end{array}\right]
$$

By inverting eq. (43) at each harmonic, approximations for $\mathbf{f}_{\mathbf{v}_{\mathbf{c}}}^{(s)}, \mathbf{f}_{\mathbf{v}_{\mathbf{S}}}^{(s)}$ denoted by $\left.\mathbf{f}_{\mathbf{v}_{\mathbf{c}}}^{(s)}\right|_{\mathbf{a}},\left.\mathbf{f}_{\mathbf{v}_{\mathbf{S}}}^{(s)}\right|_{\mathbf{a}}$ are obtained:

$$
\left[\begin{array}{l}
\left.\mathbf{f}_{\mathbf{v}_{\mathbf{C}}}^{(s)}\right|_{\mathrm{a}} \\
\left.\mathbf{f}_{\mathbf{v}_{\mathbf{S}}}^{(s)}\right|_{\mathrm{a}}
\end{array}\right]=-\left[\begin{array}{cc}
-\mathbf{M}_{\mathbf{p}_{\mathbf{1}} \mathbf{B}}(s \Omega) & \mathbf{0} \\
\mathbf{0} & \mathbf{M}_{\mathbf{p}_{\mathbf{1}} \mathbf{B}}(s \Omega) \\
-\mathbf{M}_{\mathbf{p}_{\mathbf{2}} \mathbf{B}}(s \Omega) & \mathbf{0} \\
\mathbf{0} & \mathbf{M}_{\mathbf{p}_{\mathbf{2}} \mathbf{B}}(s \Omega)
\end{array}\right]^{-\mathbf{1}}\left[\begin{array}{c}
\mathbf{z}_{\mathbf{p}_{1 \mathrm{~S}}}^{(s)} \\
\mathbf{z}_{\mathbf{p}_{\mathbf{C}}}^{(s)} \\
\mathbf{z}_{\mathbf{p}_{\mathbf{S}}}^{(s)} \\
\mathbf{z}_{\mathbf{p}_{\mathbf{6}}}^{(s)}
\end{array}\right], \quad s=1 \ldots \ldots m
$$

These are used to obtain an approximation $\left.{ }^{\mathrm{d}} \mathbf{f}_{\mathbf{v}}(t)\right|_{\mathrm{a}}$ of the periodic time history of ${ }^{\mathrm{d}} \mathbf{f}_{\mathbf{v}}(t)$, which denotes the dynamic part of $\mathbf{f}_{\mathbf{v}}(t)$ i.e. excluding the mean term in eq. (14):

$$
\left.{ }^{\mathrm{d}} \mathbf{f}_{\mathbf{v}}(t)\right|_{\mathbf{a}}=\left.\sum_{s=1}^{m} \mathbf{f}_{\mathbf{v}_{\mathbf{c}}}^{(s)}\right|_{\mathbf{a}} \cos (s \Omega t)+\left.\mathbf{f}_{\mathbf{v}_{\mathrm{S}}}^{(s)}\right|_{\mathbf{a}} \sin (s \Omega t)
$$

$\left.{ }^{d} \mathbf{f}_{\mathbf{v}}(t)\right|_{\mathrm{a}}$ contains the dynamic parts of the time histories of the $x, y$ forces at the two SFDs:

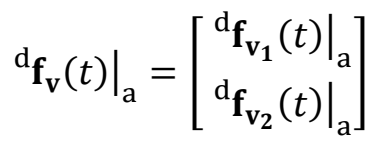


It is noted that ${ }^{d}(\quad)$ denotes the exclusion of the mean component from the quantity $(\quad$ ) (as in eq. (48)), whereas ( $)\left.\right|_{\mathrm{a}}$ denotes an approximation of the quantity ( ) based on the approximate expression of eq. (47). As shown in Section 4, for each SFD no. $n$, a trained RNN can be used so as to obtain a prediction $\left.\breve{\mathbf{v}}_{n}(t)\right|_{\mathrm{a}}$ for the periodic time history of the $2 \times 1$ vector $\left.\mathbf{v}_{n}(t)\right|_{\text {a }}$ of the $x, y$ displacements of $\mathrm{J}_{n}$ relative to $\mathrm{B}_{n}$, corresponding to a given input $\left.{ }^{d} \mathbf{f}_{\mathbf{v}_{n}}(t)\right|_{\mathrm{a}}$. From these time histories, approximations for the Fourier coefficients of the relative displacements at the SFDs, as required by eqs. $(41,42)$, can be computed by Fast Fourier Transform (FFT). The whole process is illustrated in Fig. 7.

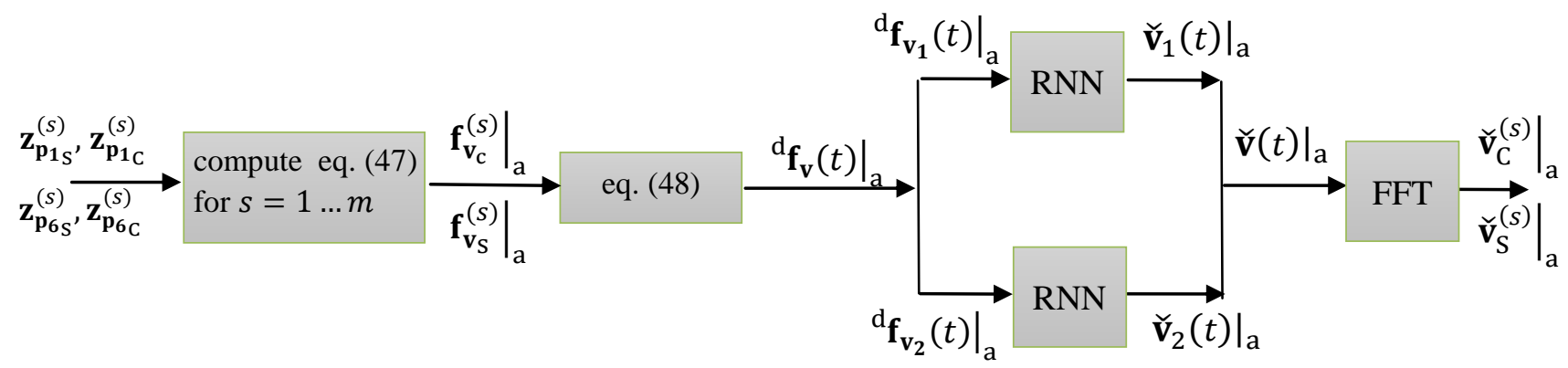

Fig. 7. Estimation of the Fourier coefficients of the journal vibration at the two SFDs from casing vibration readings at two reference locations $\left(\mathrm{P}_{1}\right.$ and $\left.\mathrm{P}_{6}\right)$

With reference to the process in Fig.7, the approximation $\left.\tilde{\mathbf{z}}(\Omega)\right|_{\mathrm{a}}$ to $\tilde{\mathbf{z}}(\Omega)$ in eqs. $(41,42)$ is given by the expression:

$$
\left.\tilde{\mathbf{z}}(\Omega)\right|_{\mathrm{a}}=\left[\begin{array}{l}
\left.\check{\mathbf{v}}_{\mathrm{C}}^{(1)}\right|_{\mathrm{a}} \\
\left.\check{\mathbf{v}}_{\mathrm{S}}^{(1)}\right|_{\mathrm{a}}
\end{array}\right]-\widetilde{\mathbf{A}}(\Omega)\left[\begin{array}{c}
\left.\mathbf{f}_{\mathbf{v}_{\mathbf{c}}}^{(1)}\right|_{\mathrm{a}} \\
\left.\mathbf{f}_{\mathbf{v}_{\mathbf{S}}}^{(1)}\right|_{\mathrm{a}}
\end{array}\right]
$$

\subsubsection{Enhanced explicit inverse problem, balancing solution}

The enhanced condition explicit inverse problem proposed in this paper is obtained by assembling Eqs. (30) and (41). The resulting expression can then be written as: 


$$
\breve{\mathbf{z}}(\Omega)=\breve{\mathbf{U}}(\Omega)\left[\begin{array}{l}
\widehat{\mathbf{u}}_{\mathrm{C}} \\
\widehat{\mathbf{u}}_{\mathrm{S}}
\end{array}\right]
$$

where

$$
\breve{\mathbf{z}}(\Omega)=\left[\begin{array}{c}
\mathbf{z}(\Omega) \\
\left.\tilde{\mathbf{z}}(\Omega)\right|_{\mathrm{a}}
\end{array}\right], \quad \breve{\mathbf{U}}(\Omega)=\left[\begin{array}{c}
\mathbf{U}(\Omega) \\
\widetilde{\mathbf{U}}(\Omega)
\end{array}\right]
$$

where $\mathbf{z}(\Omega), \mathbf{U}(\Omega)$ are the explicit inverse components that were defined by eqs. (31) and (35) respectively in section 3.2.1 and $\left.\tilde{\mathbf{z}}(\Omega)\right|_{\mathrm{a}}, \widetilde{\mathbf{U}}(\Omega)$ are the enhancements provided by the implicit inverse problem (section 3.2.2) defined by eqs. (50) and (35) respectively.

Therefore, eq. (51) defines the explicit-implicit inverse operator at one operational speed $\Omega$. As in [3], eq. (51) can be augmented over a set of operating speeds $\Omega_{k}, k=1 \ldots n_{\mathrm{o}}$.

$$
\breve{\mathbf{z}}_{\text {aug }}=\breve{\mathbf{U}}_{\text {aug }}\left[\begin{array}{l}
\widehat{\mathbf{u}}_{\mathrm{C}} \\
\widehat{\mathbf{u}}_{\mathrm{S}}
\end{array}\right]
$$

where

$$
\breve{\mathbf{z}}_{\text {aug }}=\left[\begin{array}{c}
\vdots \\
\breve{\mathbf{z}}\left(\Omega_{k}\right) \\
\vdots
\end{array}\right], \quad \breve{\mathbf{U}}_{\mathrm{aug}}=\left[\begin{array}{c}
\vdots \\
\breve{\mathbf{U}}\left(\Omega_{k}\right) \\
\vdots
\end{array}\right]
$$

and $\breve{\mathbf{z}}\left(\Omega_{k}\right), \breve{\mathbf{U}}\left(\Omega_{k}\right)$ are calculated from eqs. (52a,b).

The elements of $\widehat{\mathbf{u}}_{\mathrm{C}}, \widehat{\mathbf{u}}_{\mathrm{S}}$ can be identified through the least-squares fit solution:

$$
\left[\begin{array}{l}
\widehat{\mathbf{u}}_{\mathrm{C}} \\
\widehat{\mathbf{u}}_{\mathrm{S}}
\end{array}\right]=\breve{\mathbf{U}}_{\text {aug }}{ }^{+} \breve{\mathbf{z}}_{\text {aug }}
$$

where $\breve{\mathbf{U}}_{\text {aug }}{ }^{+}$denotes the pseudo-inverse of $\breve{\mathbf{U}}_{\text {aug. }}$. The pseudo-inverse can be obtained using the Moore-Penrose pseudo-inverse expression [27]:

$$
\breve{\mathbf{U}}_{\text {aug }}^{+}=\left(\breve{\mathbf{U}}_{\text {aug }}{ }^{\mathrm{T}} \breve{\mathbf{U}}_{\mathrm{aug}}\right)^{-\mathbf{1}} \breve{\mathbf{U}}_{\mathrm{aug}}^{\mathrm{T}}
$$


The equivalent unbalances (Fig.5) can be obtained from the identified elements of $\widehat{\mathbf{u}}_{\mathrm{C}}, \widehat{\mathbf{u}}_{\mathrm{S}}$ :

$$
\widehat{\mathbf{u}}_{\mathrm{S}}=\left[\begin{array}{l}
\hat{u}_{\mathrm{S}_{1}} \\
\hat{u}_{\mathrm{S}_{2}} \\
\hat{u}_{\mathrm{S}_{3}} \\
\hat{u}_{\mathrm{S}_{4}} \\
\hat{u}_{\mathrm{S}_{5}} \\
\hat{u}_{\mathrm{S}_{6}}
\end{array}\right]=\left[\begin{array}{c}
\widehat{U}_{1} \cos \left(\gamma+\hat{\phi}_{1}\right) \\
\widehat{U}_{1} \sin \left(\gamma+\hat{\phi}_{1}\right) \\
\widehat{U}_{2} \cos \left(\gamma+\hat{\phi}_{2}\right) \\
\widehat{U}_{2} \sin \left(\gamma+\hat{\phi}_{2}\right) \\
\widehat{U}_{3} \cos \left(\gamma+\hat{\phi}_{3}\right) \\
\widehat{U}_{3} \sin \left(\gamma+\hat{\phi}_{3}\right)
\end{array}\right] \quad \widehat{\mathbf{u}}_{\mathrm{C}}=\left[\begin{array}{c}
\hat{u}_{\mathrm{C}_{1}} \\
\hat{u}_{\mathrm{C}_{2}} \\
\widehat{u}_{\mathrm{C}_{3}} \\
\hat{u}_{\mathrm{C}_{4}} \\
\hat{u}_{\mathrm{C}_{5}} \\
\hat{u}_{\mathrm{C}_{6}}
\end{array}\right]=\left[\begin{array}{c}
\widehat{U}_{1} \sin \left(\gamma+\widehat{\phi}_{1}\right) \\
-\widehat{U}_{1} \cos \left(\gamma+\widehat{\phi}_{1}\right) \\
\widehat{U}_{2} \sin \left(\gamma+\hat{\phi}_{2}\right) \\
-\widehat{U}_{2} \cos \left(\gamma+\widehat{\phi}_{2}\right) \\
\widehat{U}_{3} \sin \left(\gamma+\hat{\phi}_{3}\right) \\
-\widehat{U}_{3} \cos \left(\gamma+\widehat{\phi}_{3}\right)
\end{array}\right]
$$

As observed in [3], eqs. $(57(a, b))$ give two possibilities for estimating the equivalent unbalance $\widehat{U}_{k}, k=1,2,3$ and its angular displacement $\widehat{\phi}_{k}$ relative to the reference phasor (Fig. 5(a)), using either $\widehat{\mathbf{u}}_{\mathrm{S}}$ (i.e. eq. (57(a))

$$
\widehat{U}_{k}=\sqrt{\left(\hat{u}_{\mathrm{S}_{2 k-1}}\right)^{2}+\left(\hat{u}_{\mathrm{S}_{2 k}}\right)^{2}} \quad,\left\{\begin{array}{l}
\cos \left(\gamma+\hat{\phi}_{k}\right)=\hat{u}_{\mathrm{S}_{2 k-1}} / \widehat{U}_{k} \\
\sin \left(\gamma+\widehat{\phi}_{k}\right)=\hat{u}_{\mathrm{S}_{2 k}} / \widehat{U}_{k}
\end{array}\right.
$$

or $\widehat{\mathbf{u}}_{\mathrm{C}}$ (i.e. eq. $(57(\mathrm{~b}))$

$$
\widehat{U}_{k}=\sqrt{\left(\hat{u}_{\mathrm{C}_{2 k-1}}\right)^{2}+\left(\hat{u}_{\mathrm{C}_{2 k}}\right)^{2}} \quad,\left\{\begin{array}{c}
\sin \left(\gamma+\hat{\phi}_{k}\right)=\hat{u}_{\mathrm{C}_{2 k-1}} / \widehat{U}_{k} \\
\cos \left(\gamma+\widehat{\phi}_{k}\right)=-\hat{u}_{\mathrm{C}_{2 k}} / \widehat{U}_{k}
\end{array}\right.
$$

The balancing force vector $\mathbf{f}_{\mathbf{b}}$, comprising forces with phasors equal and opposite to the estimated equivalent unbalance forces (Fig. 5), can be structured as follows:

$$
\mathbf{f}_{\mathbf{b}}=-\mathbf{f}_{\widehat{\mathbf{u}}}=\left[\begin{array}{c}
\Omega^{2} \widehat{U}_{1} \sin \left(\Omega t+\gamma+\left(\widehat{\phi}_{1}+\pi\right)\right) \\
-\Omega^{2} \widehat{U}_{1} \cos \left(\Omega t+\gamma+\left(\widehat{\phi}_{1}+\pi\right)\right) \\
\Omega^{2} \widehat{U}_{2} \sin \left(\Omega t+\gamma+\left(\widehat{\phi}_{2}+\pi\right)\right) \\
-\Omega^{2} \widehat{U}_{2} \cos \left(\Omega t+\gamma+\left(\widehat{\phi}_{2}+\pi\right)\right) \\
\Omega^{2} \widehat{U}_{3} \sin \left(\Omega t+\gamma+\left(\widehat{\phi}_{3}+\pi\right)\right) \\
-\Omega^{2} \widehat{U}_{3} \cos \left(\Omega t+\gamma+\left(\widehat{\phi}_{3}+\pi\right)\right)
\end{array}\right]
$$


These balancing forces are added to the actual unbalanced system in Figure 4(b), to yield the "balanced" system in Figure 8.

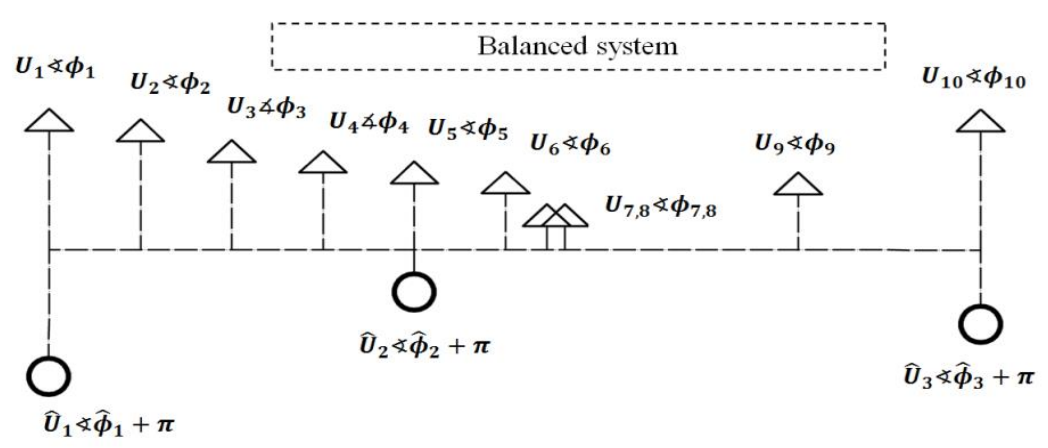

Fig. 8. "Balanced" system [3].

\section{RNN inverse SFD models.}

As observed in [17], unlike the forward model of the SFD (where the inputs are the Cartesian displacements/velocities, and the output force), it is not possible to determine the instantaneous shaft motion simply from the instantaneous SFD force. However, it is possible to determine the current value of the relative shaft displacement (output) given the time history of the SFD force (input) and previous values of the output (feedback). Hence, a time series modelling scheme based on the Nonlinear Autoregressive Exogenous (NARX) scheme [28], which accounts for system memory, is used. Such a scheme can be implemented using a recurrent neural network (RNN). The generic architecture of each of the two RNNs considered for the sprung and unsprung SFDs is illustrated in Fig. 9. The input is the SFD dynamic force signal ${ }^{\mathrm{d}} \mathbf{f}_{\mathbf{v}_{n}}\left(t_{k}\right)$ where $t_{k}=(k-1) \Delta, k=1,2, \ldots$, denotes discrete times, $\Delta$ being the sampling time resolution. Notice that, in contrast to [17], the mean is excluded since the SFD force input since this is derived from casing vibration readings as per Figure 7. Also note that the subscript "a" is dropped from the following notation for the sake of clarity. The network's output estimate for $\mathbf{v}_{n}\left(t_{k}\right)$ is denoted by $\breve{\mathbf{v}}_{n}\left(t_{k}\right)$. The inputs and outputs are normalized for network use to a range of [-1,1] [28] (where $\widetilde{(~)}$ denotes the network- 
normalised version of the variable $(\quad)$ ). System memory is taken into account through the tapped delay lines (TDLs) which are channels containing past values of the input and output (feedback) signals.

With reference to Figure 9, let $S^{(j)}$ be the number of neurons in the $j^{\text {th }}$ layer, $J$ be the total number of layers and $M_{1}$, $M_{2}$ be the numbers of delays in TDL no. 1 and TDL no.2 respectively. If $\mathbf{a}_{k}^{(j)}$ is the $S^{(j)} \times 1$ vector comprising the signal outputs of the $j^{\text {th }}$ layer, then [17]:

$$
\begin{aligned}
& \mathbf{a}_{k}^{(J)}=\widetilde{\mathbf{v}}_{n}\left(t_{k}\right) \\
& \mathbf{a}_{k}^{(1)}=\mathbf{g}^{(1)}\left(\mathbf{W}^{(1)} \mathbf{p}_{k}+\mathbf{b}^{(1)}\right) \\
& \mathbf{a}_{k}^{(j)}=\mathbf{g}^{(j)}\left(\mathbf{W}^{(j)} \mathbf{a}_{k}^{(j-1)}+\mathbf{b}^{(j)}\right), j=2, \ldots J
\end{aligned}
$$

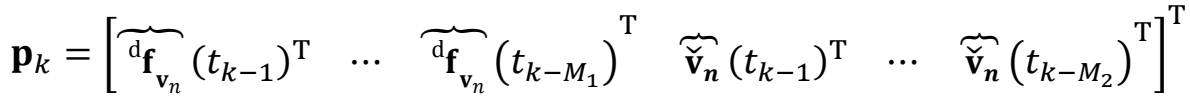

$$
\begin{aligned}
& \widetilde{\mathrm{d}}_{\mathbf{f}_{\mathbf{v}_{n}}}\left(t_{k-m}\right), \widetilde{\mathbf{v}_{n}}\left(t_{k-m}\right)=\mathbf{0}, \quad t_{k-m}<t_{1}, m=1, \ldots . \max \left(M_{1}, M_{2}\right)
\end{aligned}
$$

where $\mathbf{W}^{(j)}$ and $\mathbf{b}^{(j)}$ are respectively the matrix of weights and vector of biases of the $j^{\text {th }}$ layer and $\mathbf{g}^{(j)}(\quad)$ is a vector operator comprising the transfer functions of the neurons of the $j^{\text {th }}$ layer.

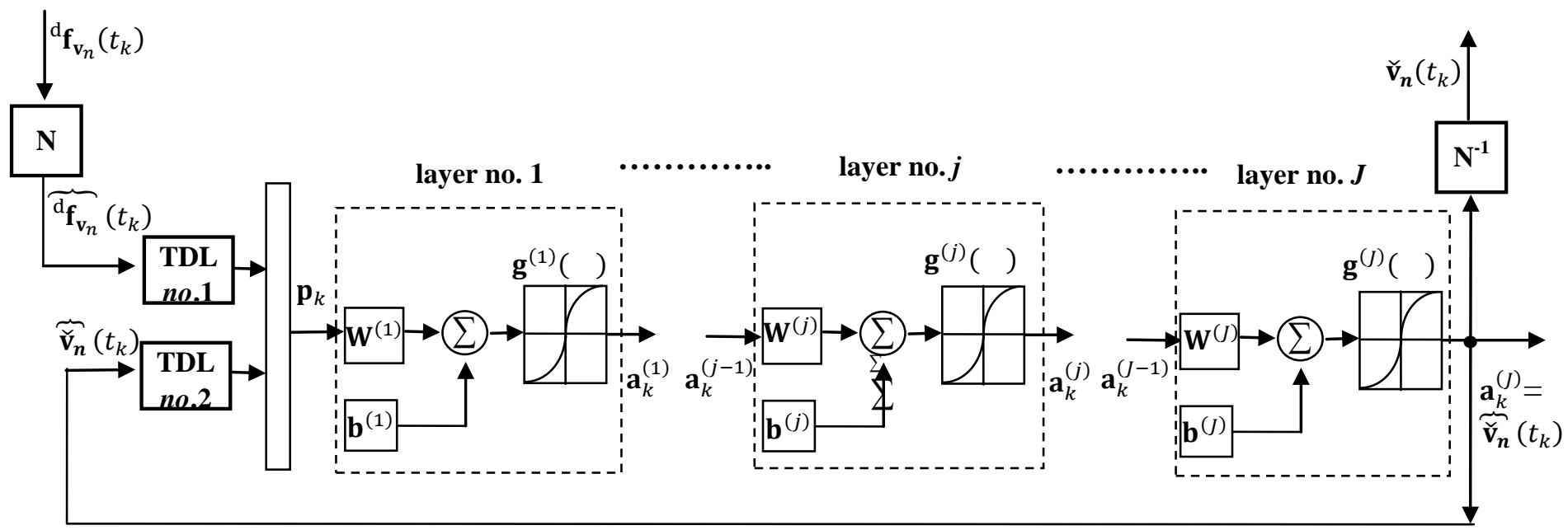

Fig. 9. RNN architecture for inverse SFD bearing model

The RNN for the inverse model each of SFD in Fig. 2 was implemented in a general purpose technical computing software that has a neural network facility [28]. For a given user-prescribed architecture, the RNN was subjected to 
input/output training data. The training procedure used the Levenberg-Marquardt optimization method [28] to determine the optimal weights and biases that minimise the mean square value of the error (difference) $\delta$ between the output of the network $\check{\mathbf{v}}_{n}\left(t_{k}\right)$ and the actual data (i.e. target) output $\mathbf{v}_{n}\left(t_{k}\right)$ for a given input ${ }^{d} \mathbf{f}_{\mathbf{v}_{n}}\left(t_{k}\right)$.

\subsection{Generation of input/output training data and RNN details}

The training data is generated by replacing the unbalance excitation in Fig. 2 by "circular chirp excitation" applied to the rotor at two locations, as shown in Fig 10. The circular chirp excitation $[21,22]$ at each location $i=1,2$ consists of a pair of forces $P_{x_{i}}(t), P_{y_{i}}(t)$ in the $x$ and $y$ directions, with time histories in the form of orthogonally-phased harmonic functions of equal and constant amplitude and steadily-increasing frequency (chirp signals):

$$
P_{x_{i}}(t)=A \sin \left[2 \pi\left\{f_{\text {init }} t+\frac{\left(f_{\text {end }}-f_{\text {init }}\right)}{\left(t_{\text {end }}-t_{\text {init }}\right)}\left(\frac{t^{2}}{2}\right)\right\}\right], \quad P_{y_{i}}(t)=-A \cos \left[2 \pi\left\{f_{\text {init }} t+\frac{\left(f_{\text {end }}-f_{\text {init }}\right)}{\left(t_{\text {end }}-t_{\text {init }}\right)}\left(\frac{t^{2}}{2}\right)\right\}\right]
$$

where $f_{\text {init }}, f_{\text {end }}$ are the excitation frequencies at the beginning $\left(t=t_{\text {init }}\right)$ and end $\left(t=t_{\text {end }}\right)$ respectively of the frequency sweep.

Accordingly, the forward problem described in section 3.1 was solved with unbalance excitation term $\mathbf{H}_{\mathbf{u}}{ }^{\mathrm{T}} \mathbf{f}_{\mathbf{u}}$ in eq. (1) replaced by $\mathbf{H}_{\text {exc }}{ }^{\mathrm{T}} \mathbf{f}_{\text {exc }}$ where:

$$
\mathbf{f}_{\mathbf{e x c}}=\left[\begin{array}{llll}
P_{x_{1}}(t) & P_{y_{1}}(t) & P_{x_{2}}(t) & P_{y_{2}}(t)
\end{array}\right]^{\mathrm{T}}
$$

and modal matrix $\mathbf{H}_{\text {exc }}(4 \times R)$ is the matrix whose columns $\Psi_{\mathrm{ext}}^{(r)}, r=1 \ldots R$ respectively are the eigenvectors evaluated at degrees of freedom corresponding to the locations and directions of the entries in $\mathbf{f}_{\text {exc }}$. The excitation amplitude $A$ (eqs. $(61 \mathrm{a}, \mathrm{b}))$ was set at $2500 \mathrm{~N}$ in both excitation locations over the rotor. The frequency sweep was performed over an interval of 0 to $30 \mathrm{~s}$ and covered frequencies in the range $133.33 \mathrm{~Hz}(8000 \mathrm{rpm})$ to $266.66 \mathrm{~Hz}$ (16000 rpm). The time histories of $\mathbf{v}_{n}(t)$ and $\mathbf{f}_{\mathbf{v}_{n}}(t)$ were computed at each time step as described in Section 3.1. For the purpose of creating the training data sets, the variable-step integration data were interpolated at equal time 
intervals $t=t_{k}=(k-1) \Delta$ where $k=1,2, \ldots$ The data for $\mathbf{f}_{\mathbf{v}_{n}}\left(t_{k}\right)$ over $0 \leq t_{k} \leq 30$ were then divided into blocks of a 100 sampling points each and the mean of the block removed, thus creating the required input training data ${ }^{\mathrm{d}} \mathbf{f}_{\mathbf{v}_{n}}\left(t_{k}\right)$. Notice that the mean of the target network output $\mathbf{v}_{n}\left(t_{k}\right)$ is retained. The resulting training data $\mathbf{v}_{n}\left(t_{k}\right)=$

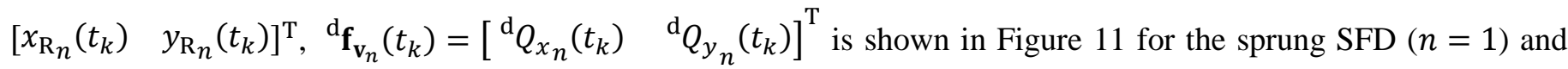
Figure 12 for the unsprung $\operatorname{SFD}(n=2)$.

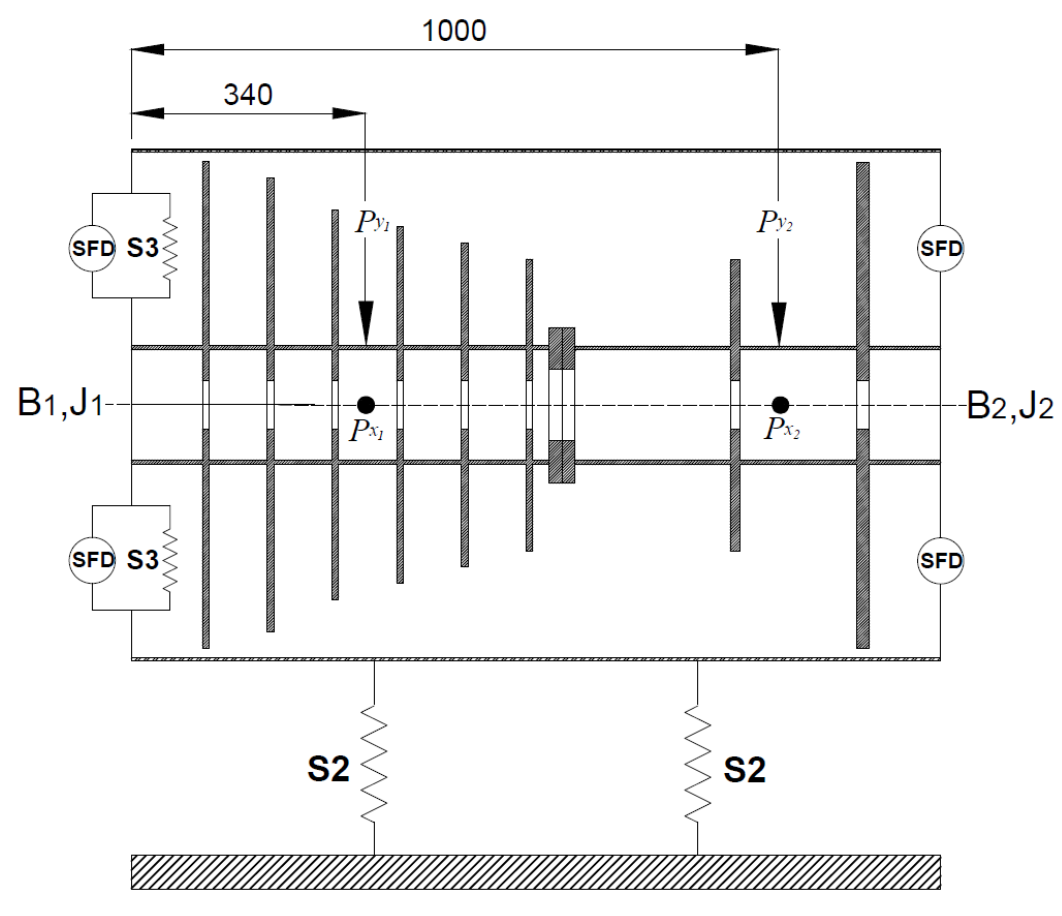

Fig. 10. Schematic description of the chirp-type excitation locations over the HP rotor (dimensions in mm). 

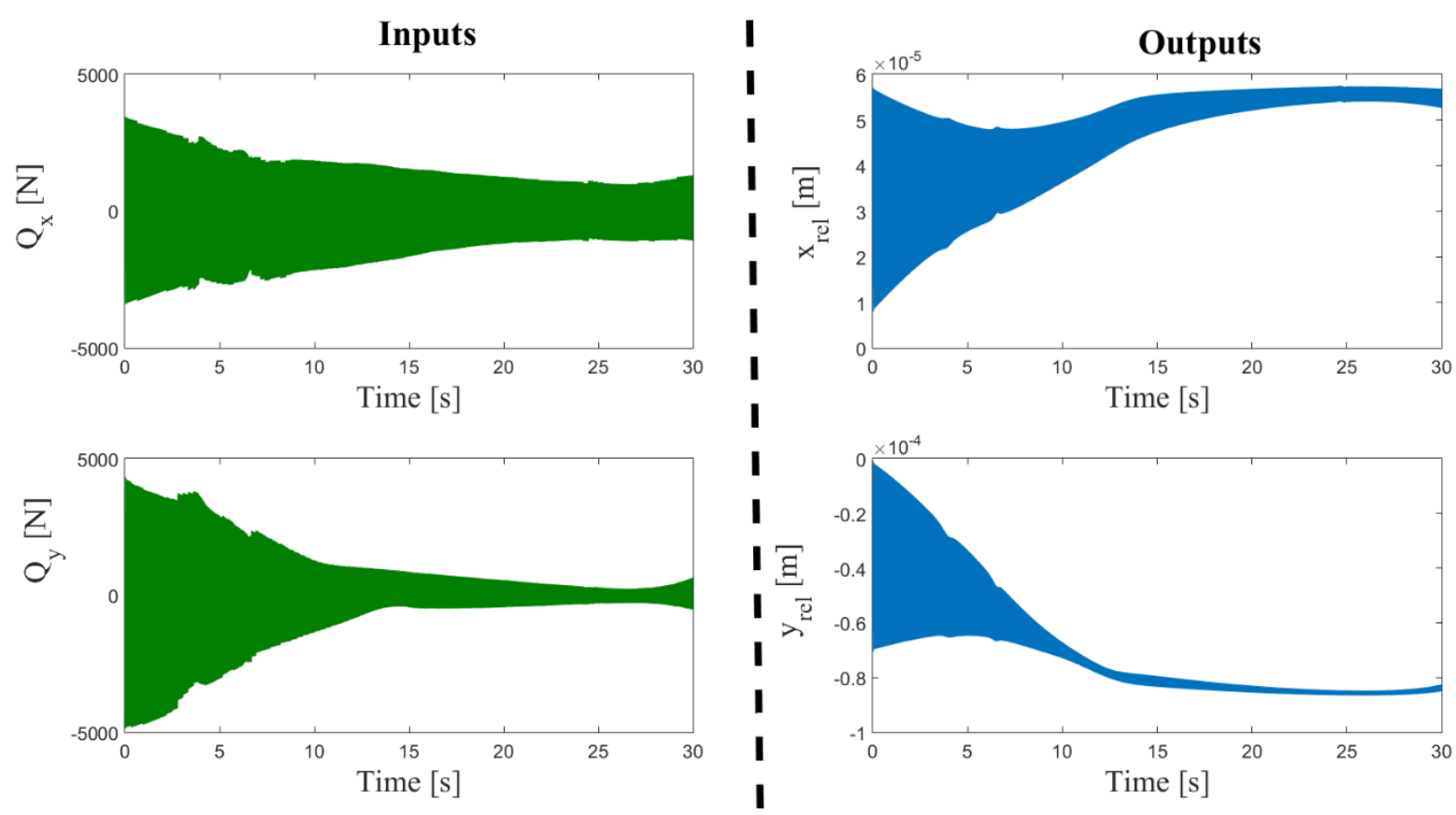

Fig. 11. Input/Output data set used to train the sprung SFD network
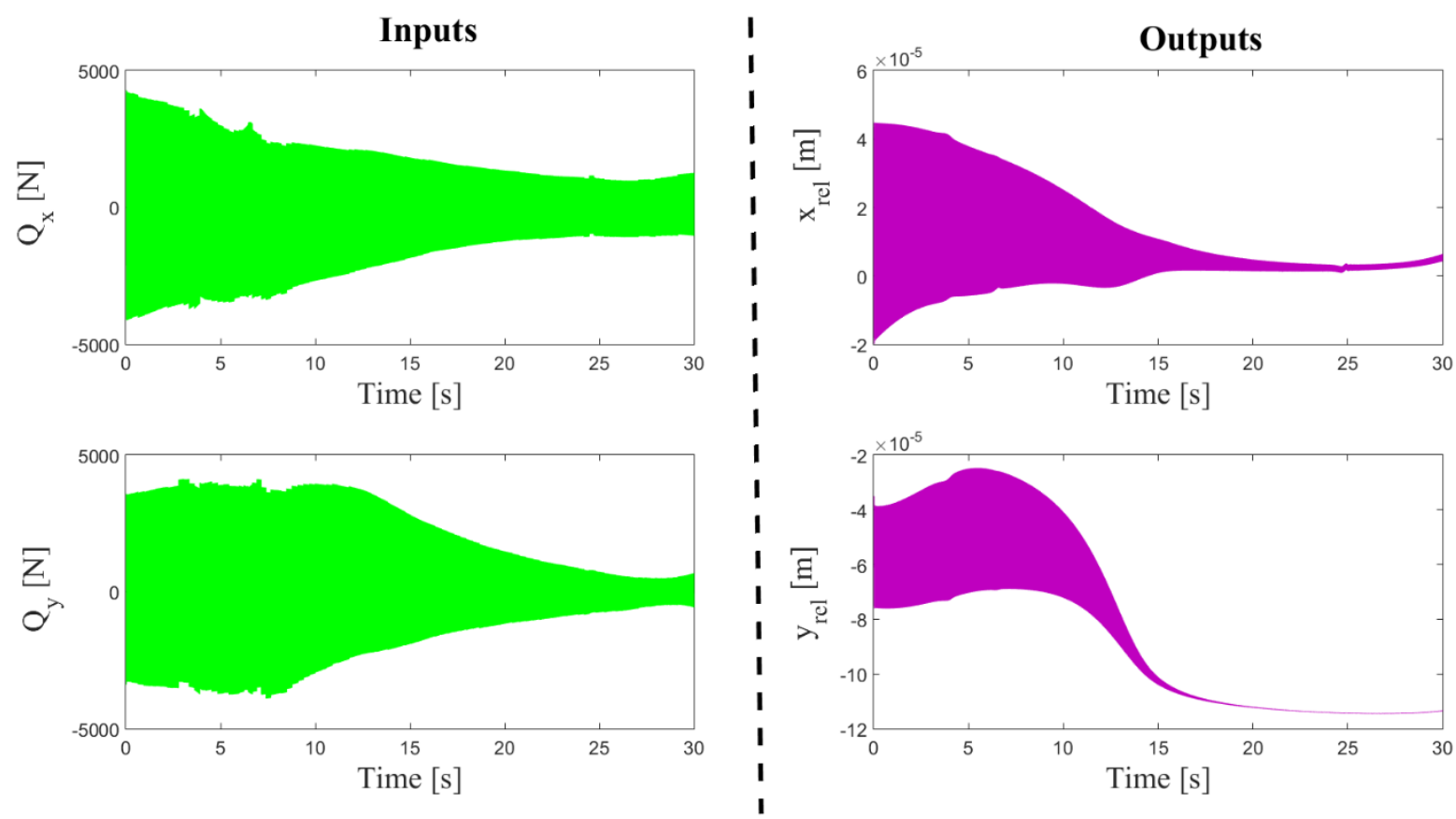

Fig. 12. Input/Output data set used to train the unsprung SFD network.

With reference to Figure 9, the sprung SFD network, trained using the data in Figure 11, had three layers with 2, 10, 2 neurons respectively and $M_{1}=2$ delays for the input and $M_{2}=2$ for the feedback. The unsprung SFD network, trained using data in Figure 12, had two layers with 4, 2 neurons respectively and $M_{1}=2$ delays for the input and 
$M_{2}=2$ for the feedback. In both cases, tan-sigmoid transfer functions $[17,28]$ were used for the neurons, except those in the final (output) layer, which used linear transfer functions.

\section{Validation Tests - Results and discussions.}

The validation tests were performed for the system in Fig. 2 subjected to the seeded unbalance distribution presented in Table 1 where the seeded unbalance planes (Fig. 4) are located at the discs in Fig. 2.

Table 1. Seeded unbalance distribution (as in [3])

\begin{tabular}{ccccccccccc}
\hline $\boldsymbol{k}$ & 1 & 2 & 3 & 4 & 5 & 6 & 7 & 8 & 9 & 10 \\
\hline $\boldsymbol{U}_{\boldsymbol{k}}\left(\times \mathbf{1 0}^{-\mathbf{3}} \mathbf{k g m}\right)$ & 0.1 & 0.5 & 0.1 & 1.0 & 0.5 & 1.0 & 0.1 & 1.5 & 0.3 & 1.5 \\
$\boldsymbol{\phi}_{\boldsymbol{k}} \times \mathbf{1 8 0} / \boldsymbol{\pi}\left(^{\circ}\right)$ & 0 & 110 & 305 & 85 & 195 & 145 & 75 & 10 & 215 & 330
\end{tabular}

The data required for the validation tests were generated by solving the forward problem as described in section 3.1 with the vector $\mathbf{f}_{\mathbf{u}}$ as per eq. (4) and Table 1. Unless otherwise stated (section 5.2.1), the reference phasor probe position $\gamma$ (Fig. 4(a)) was taken to be $0^{\circ}$.

Two types of validation tests were conducted: Validation Test no.1 (section 5.1); Validation Test no.2 (section 5.2).

\subsection{Validation study no.1: RNN inverse SFD models testing.}

This preliminary test studies the accuracy of the novel methodology of estimating the vibration at the SFD journals from the external vibration readings, as portrayed in Fig.7, which enables the enhancement $\left.\tilde{\mathbf{z}}(\Omega)\right|_{\mathrm{a}}, \widetilde{\mathbf{U}}(\Omega)$ to the novel explicit inverse problem (eq. (52a,b)). This was done by comparing, for each SFD no. $n=1,2$, the signals $\left.{ }^{\mathrm{d}} \mathbf{f}_{\mathbf{v}_{n}}(t)\right|_{\mathrm{a}}$ , $\left.\check{\mathbf{v}}_{n}(t)\right|_{\mathrm{a}}$ with the "true" signals ${ }^{\mathrm{d}} \mathbf{f}_{\mathbf{v}_{n}}(t), \mathbf{v}_{n}(t)$ respectively (the latter pair are obtained from the steady-state output of the forward solution of section 3.1). 
Figures 13(a-f) refer to the sprung SFD (LH bearing in Figure 2) and thus compare the signals $\left.{ }^{\mathrm{d}} \mathbf{f}_{\mathbf{v}_{1}}(t)\right|_{\mathrm{a}},\left.\breve{\mathbf{v}}_{1}(t)\right|_{\mathrm{a}}$ with the "true" signals ${ }^{d} \mathbf{f}_{\mathbf{v}_{1}}(t), \mathbf{v}_{1}(t)$ respectively for different fixed speeds under the unbalance conditions of Table 1, which are very different from those used in training the RNNs (section 4.1). With reference to the procedure in Figure 7, the first column of each of the Figures 13(a-f) compares the $x, y$ forces within $\left.{ }^{\mathrm{d}} \mathbf{f}_{\mathbf{v}_{1}}(t)\right|_{\mathrm{a}}$ (solid lines) with the true ones within ${ }^{\mathrm{d}_{\mathbf{f}_{1}}}$ (t) (dotted lines) ( $x, y$ forces shown in upper, lower rows respectively). It is seen that the approximation of eq. (47), based on the neglect of the linear connection between casing and rotor (the retainer spring), provides a reasonable estimate of the SFD force signal, with a moderate discrepancy in amplitude for some signals. With reference to the procedure in Figure 7, the second column of each of the Figures 13(a-f) compares the $x, y$ relative displacements within $\left.\breve{\mathbf{v}}_{1}(t)\right|_{\mathrm{a}}$ which is the RNN output for the input $\left.{ }^{{ }_{1}} \mathbf{f}_{\mathbf{v}_{1}}(t)\right|_{\mathrm{a}}$, with the true relative displacement signals within $\mathbf{v}_{1}(t)$ (dotted lines) ( $x, y$ displacement time histories shown in upper, lower rows respectively). There are two versions of $\left.\breve{\mathbf{v}}_{1}(t)\right|_{\mathrm{a}}$, shown as solid lines of different colour in the second columns of Figures 13(a-f), - the raw response from the RNN and the "FFT-response" from the RNN that is obtained by the application of FFT to the last ten cycles of the raw response and reconstruction of the time history from the resulting Fourier coefficients as per equation (15). The raw network response is seen to have an initial transient due to the default initial conditions of the RNN (eq. $(60 \mathrm{e}, \mathrm{f})$ ). However, these rapidly disappear and the subsequent steady-state raw response is in virtual perfect agreement with the FFT response. The following observations can be made when comparing the time histories of $\left.\breve{\mathbf{v}}_{1}(t)\right|_{\mathrm{a}}$ and $\mathbf{v}_{1}(t)$ :

1. The RNN-predictions correctly track the fundamental frequencies of the corresponding true signals.

2. The main discrepancy is in the mean value of the time histories of $\left.\breve{\mathbf{v}}_{1}(t)\right|_{\mathrm{a}}$ and $\mathbf{v}_{1}(t)$.

3. The correlation is reasonably good with regard to the amplitude.

It is important to note that the discrepancy in the mean is not relevant to the unbalance identification and balancing process (to be validated in the following section) since the method of section 3.2 only employs the dynamic blocks of the RHBM equations.

The discrepancies in the RNN-predicted and actual time histories of the vibration of the journal relative to the housing should also be viewed in the context of the SFD clearance circle (boundary). The third column of each of Figures 13(a-f) compares the RNN-predicted orbital motion of the journal centre $\mathrm{J}_{1}$ relative to the bearing housing centre $\mathrm{B}_{1}$ ( $y$ relative displacement vs $x$ relative displacement) with the true orbital motion within the clearance. It is noted that 
the raw RNN output orbits and the FFT-processed RNN output orbits are indistinguishable from each other and the dotted line of the true orbit appears as a solid line due to repeated cycling. The orbital plots show that, in the context of the clearance circle, the RRN-predicted orbits are in reasonably good agreement with the true ones in terms of size and orientation. The orbital plots also show that there is reasonably good agreement with regards to mean position when viewed within the clearance circle. However, it should be noted that the results in Figures 13(a-f) refer to the sprung SFD, for which the mean position is mainly determined by the static offset ( $\mathbf{e}_{0_{1}}$ in eq. (2), section 3.1) and this offset was used in the training. 

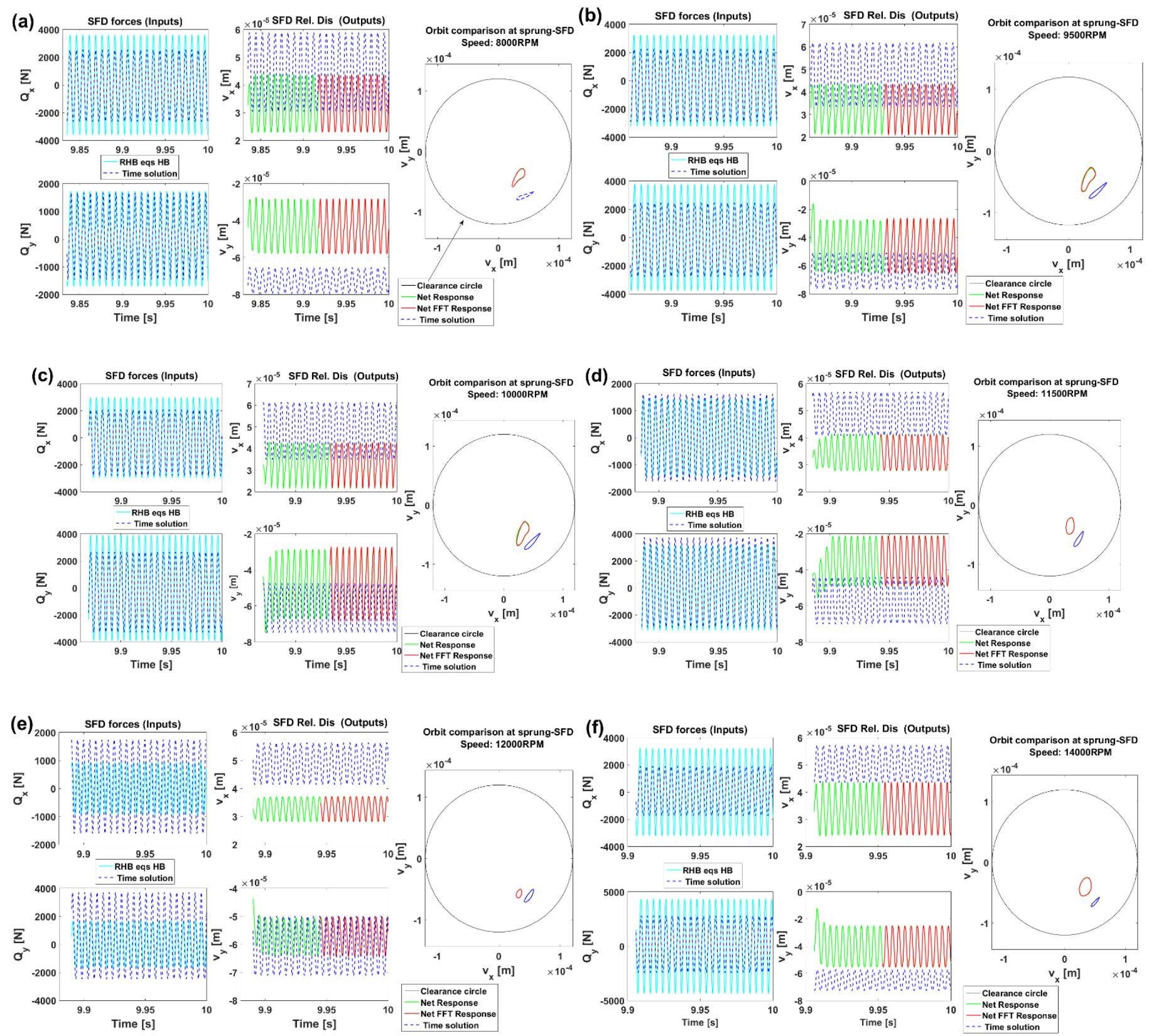

Fig. 13. Comparison of the signals $\left.{ }^{d} \mathbf{f}_{\mathbf{v}_{1}}(t)\right|_{a},\left.\breve{\mathbf{v}}_{1}(t)\right|_{\mathrm{a}}$ with the "true" signals ${ }^{\mathrm{d}} \mathbf{f}_{\mathbf{v}_{1}}(t), \mathbf{v}_{1}(t)$ at the SPRUNG SFD bearing for six distinct speeds under the unbalance conditions of Table 1 (a) $8000 \mathrm{rpm}$; (b) $9500 \mathrm{rpm}$; (c) $10000 \mathrm{rpm}$; (d) $11500 \mathrm{rpm}$; (e) $12000 \mathrm{rpm}$; (f) $14000 \mathrm{rpm}$.

Figures 14(a-f) present the corresponding results for the unsprung SFD (RH bearing in Fig. 2). With reference to the first column of each of the Figures 14(a-f) it is seen that the correlation between the $x, y$ forces within $\left.{ }^{\mathrm{d}} \mathbf{f}_{\mathbf{v}_{2}}(t)\right|_{\mathrm{a}}$ (solid lines) with the true ones within ${ }^{d} \mathbf{f}_{\mathbf{v}_{2}}(t)$ (dotted lines) is very good and much better than the previous case for the sprung SFD. This can be explained by the fact that the approximations $\left.{ }^{d} \mathbf{f}_{\mathbf{v}_{1,2}}(t)\right|_{\mathbf{a}}$ (eq. (49)), determined from eq. 
(47), are based on the neglect of the retainer spring in Fig. 2 and there is no retainer spring in the case of SFD no.2. The improved accuracy in the case of $\left.{ }^{\mathrm{d}} \mathbf{f}_{\mathbf{v}_{2}}(t)\right|_{\mathrm{a}}$, which is fed to the RNN to output the $x, y$ relative displacements within $\left.\breve{\mathbf{v}}_{2}(t)\right|_{\mathrm{a}}$, is probably a major reason why these also show improved correlation with the true signals within $\mathbf{v}_{2}(t)$, as can be seen from the second and third columns of Figures 14(a-f). From the second columns of Figures 14(a-f), one notes that the initial transient phase of the raw RNN response signals in $\left.\breve{\mathbf{v}}_{2}(t)\right|_{\mathrm{a}}$ (due to the initial conditions, eq. $(60 \mathrm{e}, \mathrm{f}))$ takes considerably longer to disappear than the previous case of the sprung SFD, especially in the case of the $y$-signals. This is because in the case of the actual unsprung (unsupported) SFD that the RNN mimics, the mean position is determined by the equilibrium between the equivalent rotor weight $J_{2}$ (section 3.1) and the mean SFD force in the $y$-direction, which is itself a function of the vibration of $\mathbf{J}_{2}$. As the transients disappear from the raw RNN response, its mean position shifts towards those of the true signals in $\mathbf{v}_{2}(t)$ (shown dotted in the second columns of Figures 14(a-f)). The third columns of Figures 14(a-f) show remarkably improved correlation between the steadystate RNN-predicted orbits and the true orbits in terms of orbit size, orientation and mean position within the clearance, relative to the previous sprung SFD case. 

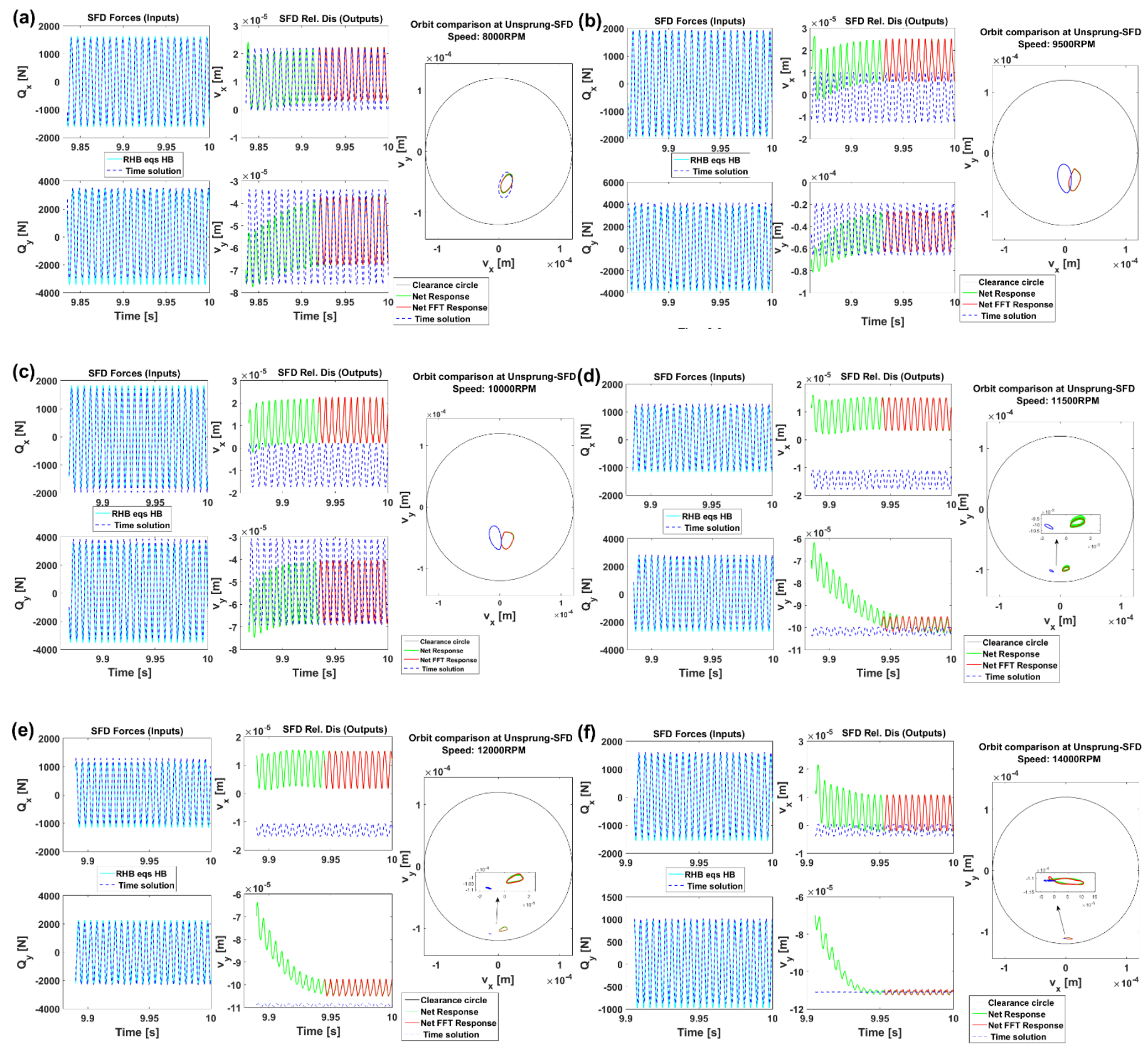

Fig. 14. Comparison of the signals $\left.{ }^{d} \mathbf{f}_{\mathbf{v}_{2}}(t)\right|_{\mathrm{a}},\left.\breve{\mathbf{v}}_{2}(t)\right|_{\mathrm{a}}$ with the "true" signals ${ }^{\mathrm{d}} \mathbf{f}_{\mathbf{v}_{2}}(t), \mathbf{v}_{2}(t)$ at the UNSPRUNG SFD bearing for six distinct speeds under the unbalance conditions of Table 1 (a) $8000 \mathrm{rpm}$; (b) $9500 \mathrm{rpm}$; (c) $10000 \mathrm{rpm}$; (d) $11500 \mathrm{rpm}$; (e) $12000 \mathrm{rpm}$; (f) $14000 \mathrm{rpm}$ (a) $8000 \mathrm{rpm}$; (b) $9500 \mathrm{rpm}$; (c) $10000 \mathrm{rpm}$; (d) $11500 \mathrm{rpm}$; (e) 12000 rpm; (f) $14000 \mathrm{rpm}$. 


\subsection{Validation study no.2: Balancing effectiveness testing under different practical cases.}

This test studies the effectiveness of the enhanced balancing solution method, as per section 3.2.3. In this test, having solved eq. (53), the equivalent unbalance magnitudes and phases $\widehat{U}_{k}, \widehat{\phi}_{k}$ in the three balancing planes (Fig. 5) are calculated from the average of the estimates provided by the alternative expressions of eqs. $(58 \mathrm{a} 1, \mathrm{a} 2)$ and $(58 \mathrm{~b} 1, \mathrm{~b} 2)$. The balancing force vector is then set up as in eq. (59) and the vibration response of the "balanced" system in Fig. 8 is determined by re-computing the forward problem with an additional term $\mathbf{H}_{\mathbf{b}}{ }^{\mathrm{T}} \mathbf{f}_{\mathbf{b}}$ on the righthand side of eq. (1), where $\mathbf{H}_{\mathbf{b}}$ is the $6 \times R$ matrix whose columns are the eigenvectors evaluated in the $x, y$ directions at the prescribed balancing locations along the rotor. As in [3], the degree of effectiveness of the balancing solution is quantified by the average percentage vibration reduction (PVR) (in terms of vibration velocity amplitudes, $\left.A_{\text {before}}, A_{\text {after }}\right)$ at each sensor location $\mathrm{P}_{i}$ over the speeds $\Omega_{k}\left(k=1 \ldots n_{\mathrm{o}}\right)$ at which the vibration readings were taken:

$$
\operatorname{PVR}\left(\mathrm{P}_{i}\right)=\left.\overline{\left(\frac{A_{\text {before }}-A_{\text {after }}}{A_{\text {before }}}\right)}\right|_{\Omega_{k}, k=1 \ldots n_{\mathrm{o}}} \times 100
$$

With reference to the procedure in section 3.2 , the speeds $\Omega_{k}$ in eqs. $(54 a, b)$ at which the unbalance vibration data were taken totalled 14 in number $(8000,8500,9000, \ldots, 14000$ and $14500 \mathrm{rpm})$ covering the typical operating speed range of the HP rotor of an aero-engine [3]. The three balancing planes (at which the equivalent unbalance was determined (Fig. 5) and balancing correction applied (Fig. 8)), were chosen to be disk nos. 1, disk 5 and disk 10 in Fig. 2 or Fig. 4, based on previous studies [1, 3].

The superiority of the enhanced explicit inverse problem method over the previously published explicit inverse problem method [3] for the system in Fig. 2 is first demonstrated (section 5.2.1). The robustness of the novel method is then studied by examining it for repeatability (section 5.2.2), different levels of noise (section 5.2.3) and uncertainty in structural model parameters (section 5.2.4). 


\subsubsection{Effect of implicit term enhancement on the explicit inverse problem.}

The main contribution of this paper is the enhancement of the inverse problem devised in [3] through the addition of the implicit inverse problem terms $\left.\widetilde{\mathbf{z}}(\Omega)\right|_{\mathrm{a}}, \widetilde{\mathbf{U}}(\Omega)$ to the matrices $\breve{\mathbf{z}}(\Omega), \breve{\mathbf{U}}(\Omega)$ (eqs. (52a,b)) that constitute the matrices $\breve{\mathbf{z}}_{\text {aug }}, \breve{\mathbf{U}}_{\text {aug }}$ of the final inverse problem for equivalent unbalance identification defined by eq. (53). Therefore, the first step is to demonstrate the effect of the enhancement on the balancing solution by solving the problem defined by eq. (53) both with and without the enhancing terms $\left.\tilde{\mathbf{z}}(\Omega)\right|_{\mathrm{a}}, \widetilde{\mathbf{U}}(\Omega)$ in eqs. (52a,b).

Figure 15 shows the vibration velocity orbits at the sensor locations ( $y$-direction vibration velocity versus $x$-direction vibration velocity) along the casing (Fig. 6) at different speeds before balancing, and after balancing with and without the enhancing terms. As observed in [3], the orbital distortion (from an ellipse) in the "before balancing" orbits is a clear indication of nonlinearity of the casing vibration response. This distortion is caused by the harmonics in the response due to the nonlinear oil-film forces from the SFDs. It is quite clear from Fig.15 that the introduction of the implicit inverse problem terms $\left.\widetilde{\mathbf{z}}(\Omega)\right|_{\mathrm{a}}, \widetilde{\mathbf{U}}(\Omega)$ to the matrices $\breve{\mathbf{z}}(\Omega), \breve{\mathbf{U}}(\Omega)$ (eqs. (52a,b)) drastically improves the balancing solution, as evident from the substantial reduction in orbit sizes. These terms are actually essential for balancing the system in Fig. 2 since Fig. 15 shows that their omission would result in the "after-balancing" vibration orbits becoming much bigger than the "before balancing" vibration orbits. Fig. 16 compares the percentage vibration reductions PVRs (eq. (63)) after balancing the system with and without the enhancing implicit terms, where VPVR is the PVR for the $y$ vibration and HPVR is the PVR for the $x$ vibration. It is evident that the omission of the enhancing terms will result in negative PVRs (amplification of vibration). 

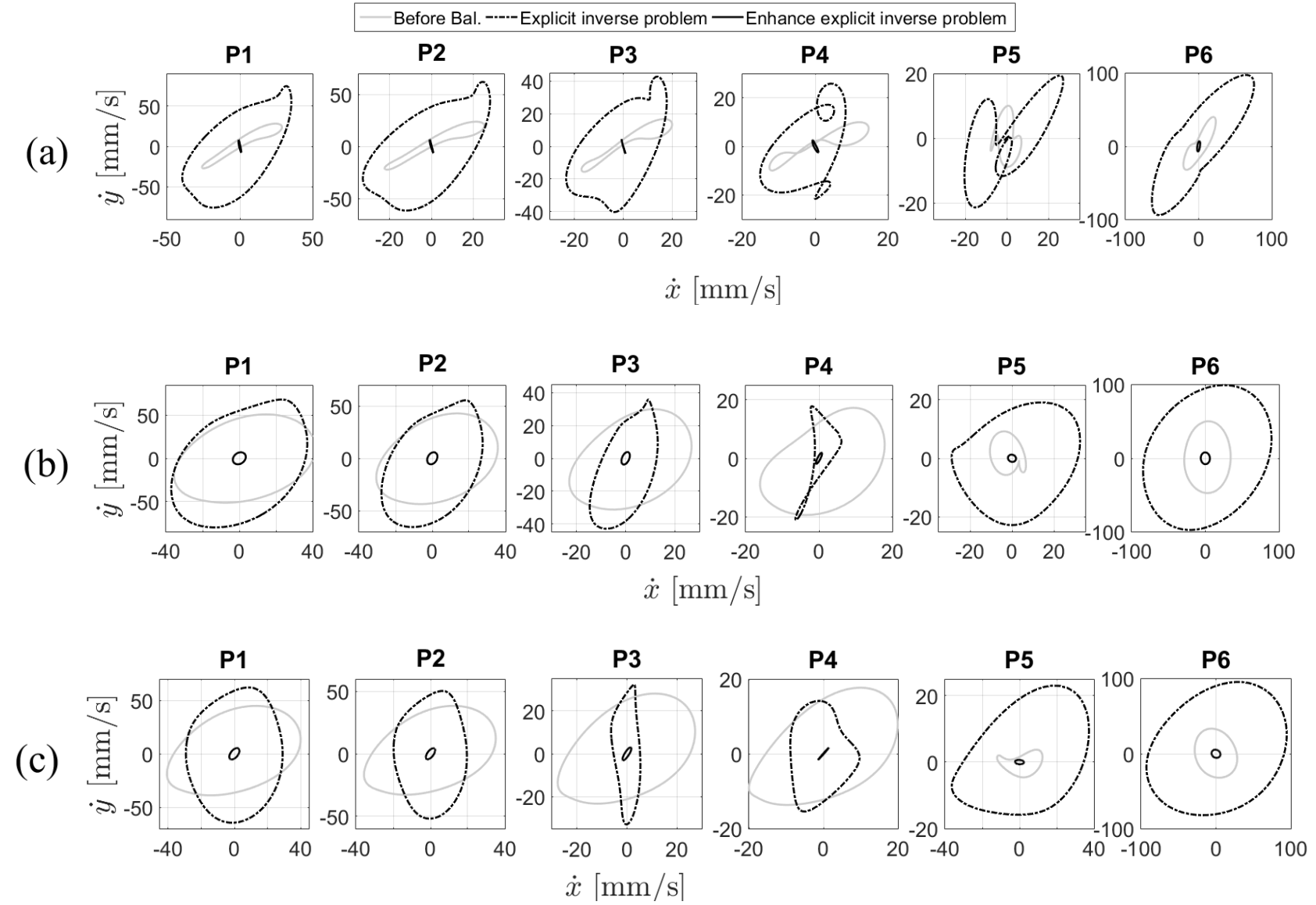

Fig. 15. Velocity orbits at casing measurement locations before balancing and after balancing using fully explicit inverse problem and enhanced explicit inverse problem (no added noise). (a) $8500 \mathrm{rpm}$; (b) $12000 \mathrm{rpm}$; (b) 14000 rpm. 

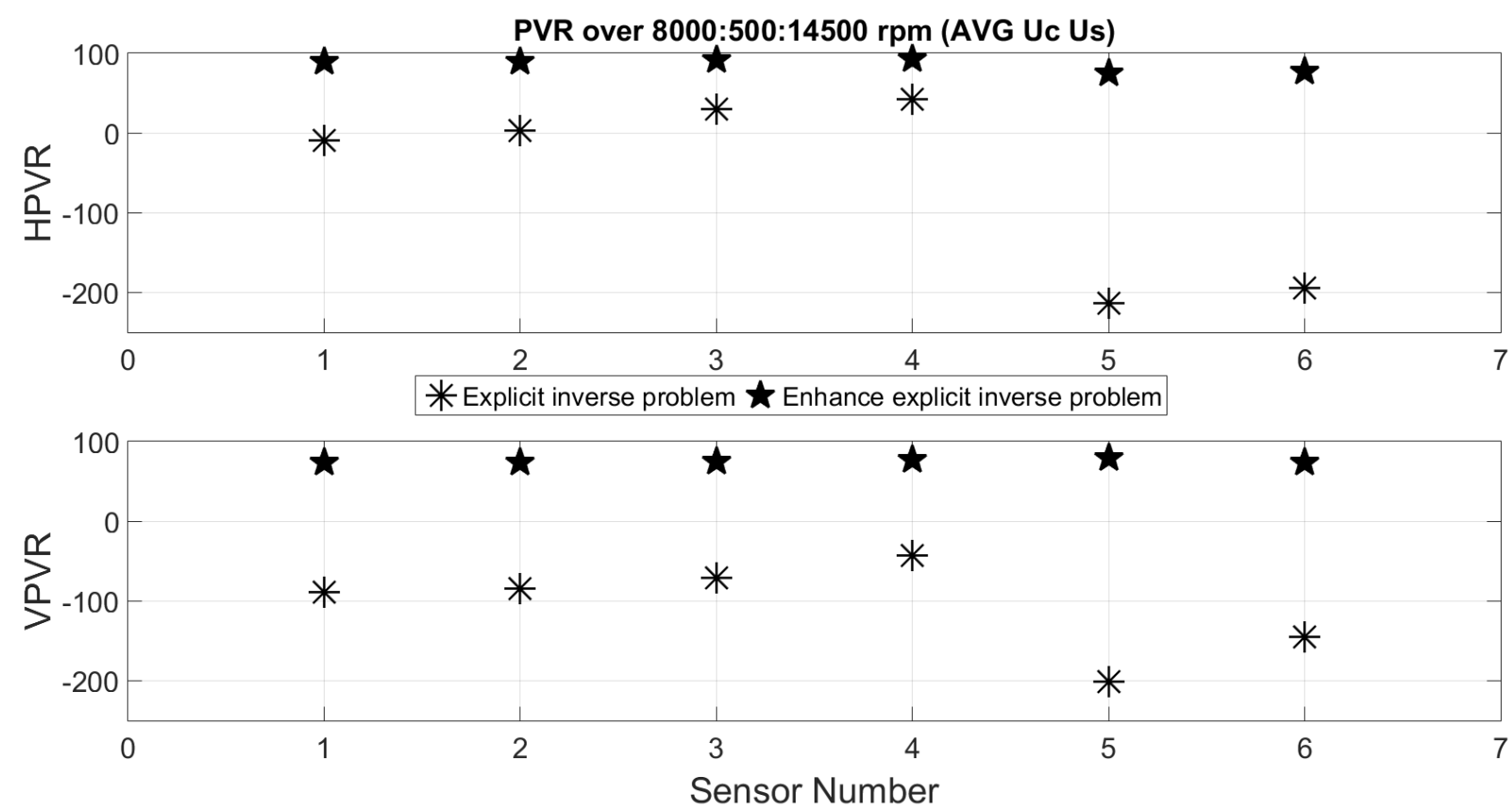

Fig. 16. Comparison of PVRs (eq. (63)) after balancing using fully explicit inverse problem and enhanced explicit inverse problem (no added noise, HPVR refers to $x$-direction, VPVR refers to $y$-direction).

The superiority of the enhanced explicit inverse problem method is attributed to the additional information on the vibration at the SFD journals that is provided by the RNN that act as virtual instruments as per methodology of Fig. 7. This information is contained in the term $\left.\tilde{\mathbf{z}}(\Omega)\right|_{\mathrm{a}}$ added to eq. (52a). Moreover, the companion term $\widetilde{\mathbf{U}}(\Omega)$, added to eq. (52b), is found to massively improve the condition of the matrix $\breve{\mathbf{U}}_{\mathrm{aug}}$ (eq. (54b)) as follows:

- condition number of $\widetilde{\mathbf{U}}_{\text {aug }}$ with $\widetilde{\mathbf{U}}(\Omega)$ omitted from eq. (52b) is 7103.1;

- condition number of $\widetilde{\mathbf{U}}_{\text {aug }}$ with $\widetilde{\mathbf{U}}(\Omega)$ included in eq. (52b) is 47.26 .

It is noted that the least-squares solution eq. (55) minimises the residual error

$$
E=\left\|\breve{\mathbf{z}}_{\text {aug }}-\breve{\mathbf{U}}_{\text {aug }}\left[\begin{array}{l}
\widehat{\mathbf{u}}_{\mathrm{C}} \\
\widehat{\mathbf{u}}_{\mathrm{S}}
\end{array}\right]\right\|
$$

in order identify the equivalent unbalance in three prescribed planes (Fig. 5(a,b)) using casing vibration data from the true unbalance distribution over ten planes (Fig. 4(a,b)). The repeatability of the solution for different positions of the stationary phase probe $\gamma$ (Fig. 4(a)), and its robustness to noise in the vibration data / uncertainties in the rotor-casing 
model, are strongly influenced by the condition of the matrix $\widetilde{\mathbf{U}}_{\text {aug }}$. Hence, the improvement of the condition of $\widetilde{\mathbf{U}}_{\text {aug }}$ through the inclusion of $\widetilde{\mathbf{U}}(\Omega)$ in eq. (52b) is also essential to guarantee the repeatability and robustness of the novel method, which shall be demonstrated in the following sections.

\subsubsection{Repeatability for different phase probe positions.}

Table 2 shows the estimates for the equivalent unbalance magnitudes and phases $\widehat{U}_{k}, \widehat{\phi}_{k}(k=1,2,3)$ for 6 different angular locations $\gamma$ of the stationary phase probe (Fig.4). It is seen that the degree in variability is low and is in fact lower than that obtained in the similar test for the simpler systems considered in [3] using the unenhanced (fully explicit) inverse problem method. It is noted that phase probe location $\gamma$ has no effect on the "before balancing" orbits (shown in Fig. 15) - in the absence of measurement noise these remain identical subject to minute differences from the error tolerance of the integrator. However, the shift in phase datum will vary the Fourier coefficients $\mathbf{z}_{\mathbf{p}_{i S}}^{(s)}, \mathbf{z}_{\mathbf{p}_{i_{\mathrm{C}}}}^{(s)}$ of the casing vibration data (eq.(8)) which are used to calculate the terms in $\breve{\mathbf{z}}_{\text {aug }}$ (eq.(53), via eq. (52a), eq. (31), Fig. 7 and eq. (50)) and thus causing the fluctuations in $\widehat{U}_{k}, \widehat{\phi}_{k}$ shown in Table 2.

Table 2. Repeatability test results for $\widehat{U}_{k}[\mathrm{~kg} \mathrm{~mm}]$ and $\widehat{\phi}_{k}[\mathrm{deg}](k=1,2,3)$ as stationary phase probe location $\gamma$ is varied (no added noise).

\begin{tabular}{ccccccccccccc}
\hline $\boldsymbol{r}$ & \multicolumn{2}{c}{$\mathbf{0}^{\circ}$} & \multicolumn{2}{c}{$\mathbf{1 0}^{\circ}$} & \multicolumn{2}{c}{$\mathbf{2 0}^{\circ}$} & \multicolumn{2}{c}{$\mathbf{3 0}^{\circ}$} & \multicolumn{2}{c}{$\mathbf{5 0}^{\circ}$} & \multicolumn{2}{c}{$\mathbf{6 0}^{\circ}$} \\
\hline$k$ & $\widehat{U}_{k}$ & $\hat{\phi}_{k}$ & $\widehat{U}_{k}$ & $\widehat{\phi}_{k}$ & $\widehat{U}_{k}$ & $\widehat{\phi}_{k}$ & $\widehat{U}_{k}$ & $\widehat{\phi}_{k}$ & $\widehat{U}_{k}$ & $\widehat{\phi}_{k}$ & $\widehat{U}_{k}$ & $\widehat{\phi}_{k}$ \\
\hline 1 & 0.47 & 107.5 & 0.47 & 107.7 & 0.47 & 107.6 & 0.47 & 107.4 & 0.47 & 106.5 & 0.47 & 106.3 \\
2 & 1.6 & 84.87 & 1.6 & 84.98 & 1.6 & 85.06 & 1.6 & 85.05 & 1.6 & 84.90 & 1.6 & 84.84 \\
3 & 1.6 & -40.3 & 1.6 & -40.90 & 1.6 & -42.03 & 1.6 & -43.27 & 1.6 & -43.99 & 1.6 & -43.13 \\
\hline
\end{tabular}

\subsubsection{Noise}

In these tests, the simulated vibration time histories from the sensors were corrupted with different levels of noise quantified by the signal-to-noise-ratio (SNR) as in [3]: 


$$
\mathrm{SNR}=20 \log _{10}\left(\frac{A_{\text {signal }}}{A_{\text {noise }}}\right)
$$

where $A_{\text {signal }}$ is the root-mean-square (RMS) value of the signal without noise and $A_{\text {noise }}$ is the RMS value of the noise.

Fig. 17 presents PVRs for the balancing solution at different levels of noise. It is seen that a reduction of SNR from $25 \mathrm{~dB}$ to $20 \mathrm{~dB}$ causes a significant reduction in the PVRs. The balancing effectiveness at $20 \mathrm{~dB}$ SNR was considerably better in [3]. However, the systems in [3] were considerably simpler and did not require the additional information from the RNN inverse SFD models. The accuracy of the latter in estimating the SFD journal relative vibration will be affected by noise in the casing vibration readings (see process in Fig. 7). Nonetheless, 25dB SNR is still a realistic measurement scenario and presents PVRs of at least $60 \%$ at all sensor locations.

Figure 18 shows the casing vibration velocity orbits at 8500,12000 and $14000 \mathrm{rpm}$ before and after balancing in the presence of $25 \mathrm{~dB}$ SNR. It is seen that the reduction in the nonlinear vibration orbits remains substantial. In the subsequent Section 5.3.4 a SNR of $25 \mathrm{~dB}$ is assumed.

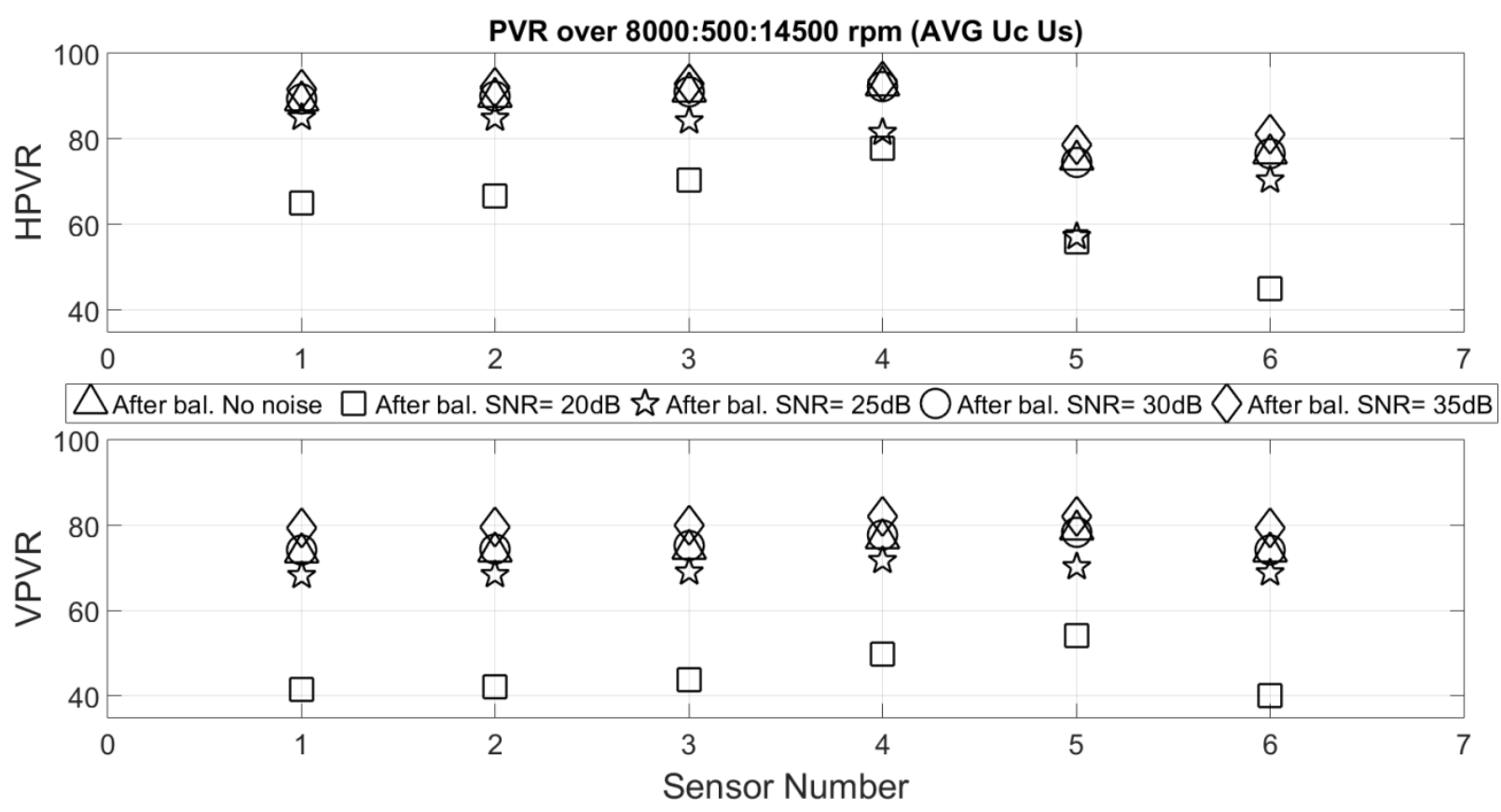

Fig. 17. PVRs for different levels of signal-to-noise (SNR) ratio for $\gamma$ at $0^{\circ}$

(HPVR refers to $x$-direction, VPVR refers to $y$-direction) 
(a)
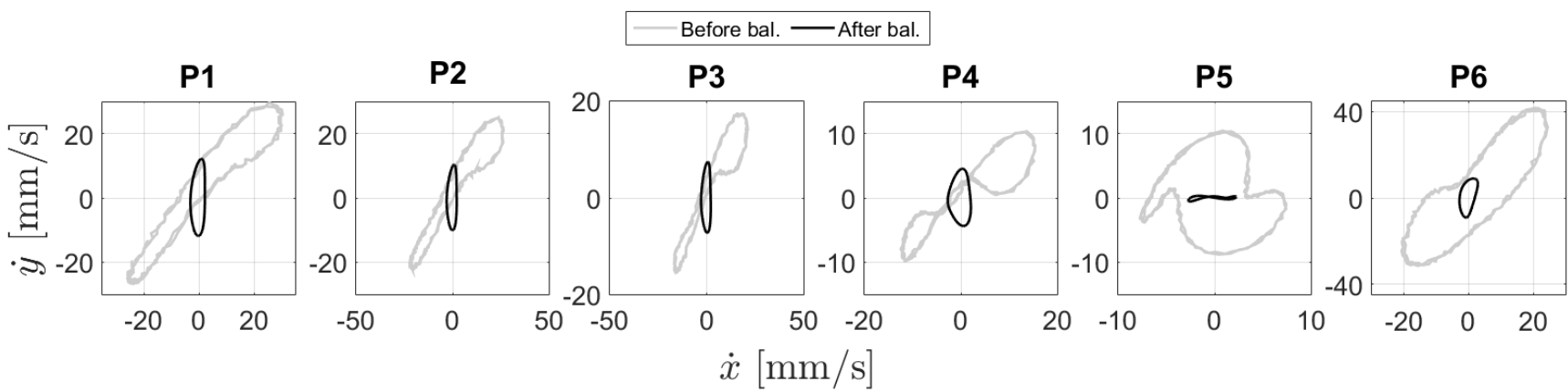

(b)
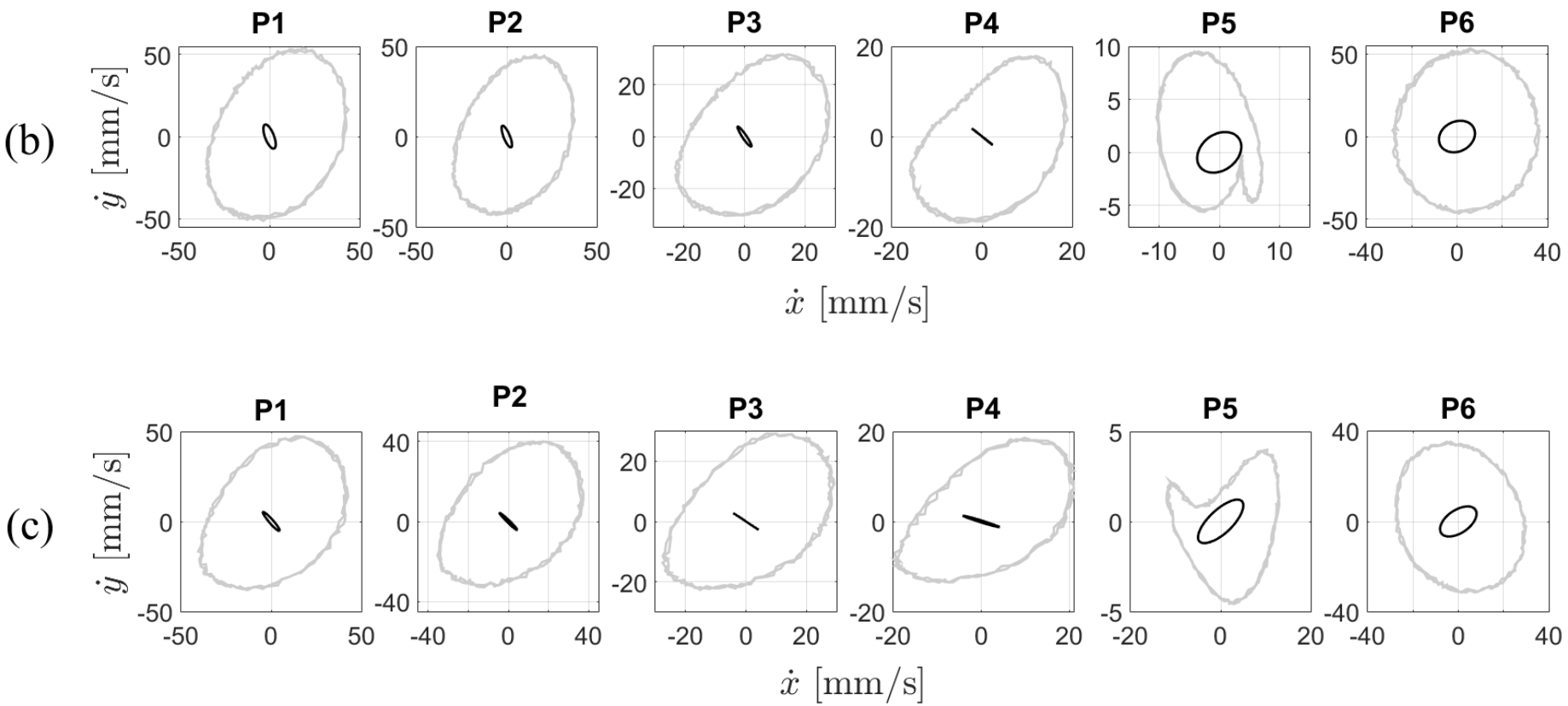

Fig. 18. Velocity orbits at casing measurement locations before balancing and after balancing ( $\mathrm{SNR}=25 \mathrm{~dB})$. (a) 8500 rpm; (b) $12000 \mathrm{rpm}$; (b) $14000 \mathrm{rpm}$

\subsubsection{Rotor-casing structural model uncertainties.}

As in all other inverse problem unbalance identification techniques that only use sensors attached to the casing (noninvasive), the effectiveness of the balancing solution is subject to uncertainties in the a priori known dynamic model of the linear part of the structure. As in [3] the robustness of the balancing process was assessed by prescribing a random error to the outer diameter of each the 10 disks (Fig. 2). This geometric random error (GRE) affects the mass and moment of inertia properties of each disk, thus affecting the eigenfrequencies and mode shapes (Fig. 3). The validation was then performed according to the following steps [3]: 
1. Generation of the vibration data (section 3.1) from the system in Fig. 4 corrupted within a certain level of GRE.

2. Solution of the inverse problem (section 3.2) using the original non-corrupted model (reference system) and the "real" vibration data obtained at step no.1.

3. Generation of the vibration data (section 3.1) from the "balanced" system in Fig. 8 corrupted with the prescribed level of GRE used in step 1.

4. Comparison of the vibration data in step nos. 1 and 3 as a PVR (eq. (63)).

Figure 19 shows the PVRs obtained under conditions of up to $\pm 2 \%$ GRE at $25 \mathrm{~dB}$ SNR. The balancing effectiveness is seen to be reasonably robust, with PVRs remaining above 50\%. The uncertainty bandwidth is seen to be marginally wider than that obtained with the simpler system and method of [3]. However, this is not unexpected since, similarly to the previous section on noise, the GRE will affect the accuracy of the necessary additional information from the RNN inverse SFD models since the process in Fig. 7 uses eq. (47).
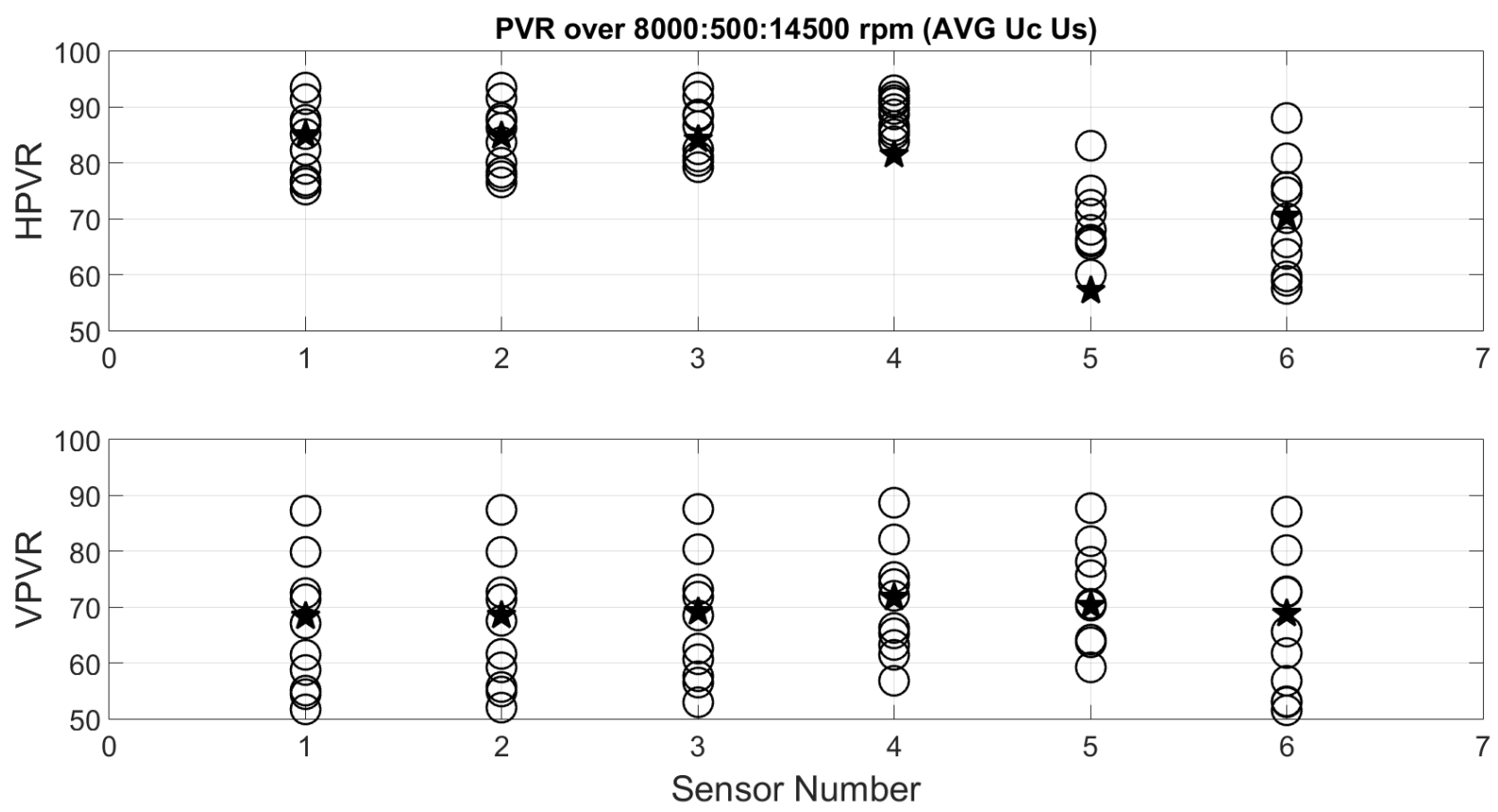

Fig. 19: Effect of rotor/casing structural modelling uncertainty - PVRs for a geometric random error (GRE) of up to $\pm 2 \%$, in the presence of noise of $25 \mathrm{~dB}$ SNR, for $\gamma$ at $0^{\circ}$ (HPVR refers to $x$-direction, VPVR refers to $y$-direction) $(\star$ no GRE; $\bigcirc:$ GRE of $\pm 2 \%$ ) 


\subsubsection{Discussion on practical implementation and uncertainty in RNN inverse SFD models.}

The aim of this paper was to introduce an effective method for balancing the previously unconsidered practical configuration in Fig. 2 and validate it using simulated casing vibration readings as done for the simpler systems in [1] and [3]. This motivates the building of a test rig for the system in Fig. 2 (referred to as test rig "A" in the following text) to validate the method under experimental conditions. Although this is outside the scope of the present paper, it is important to discuss how this next stage of the work would be implemented, particularly with regard to the RNN inverse SFD models, which are crucial to the process in Fig. 7 for the evaluation of the enhancing term $\left.\tilde{\mathbf{z}}(\Omega)\right|_{\mathrm{a}}$ in eq. (52a). The training data for the RNN inverse SFD models required by test rig A can be generated under circular chirp excitation conditions in one of the following alternative ways.

(a) Experimental testing of each bearing in isolation using a separate purpose-built test rig "B" (as used in [17] and [22]) to generate the input/output training data.

(b) Using the theoretical model of the test rig A and its bearings to generate simulated training data from the forward solution as done in this paper (section 4.1).

In either of the above cases, the extracted RNN inverse SFD models can then be used with actual casing vibration measurements of test rig A to balance it according to the method introduced in this paper.

Each identified RNN inverse model is in principle applicable for a particular set of SFD features: geometric parameters (particularly the radial clearance); type and degree of sealing; oil viscosity; degree of static offset (sprung SFD); magnitude of static load (unsprung SFD). Deviations in these parameters between training and operational scenarios introduce uncertainty in the RNN blocks in Fig. 7 which affects the quality of the balancing solution. The empirical identification approach of (a) (see above) is more labour-intensive relative to approach (b), but it reduces the uncertainty since it can capture effects that are difficult to model e.g. irregularities in the radial clearance profile [17], [22]. In the case of approach (b), the sensitivity of the balancing solution to uncertainty in the assumed radial clearance can be investigated using simulated casing vibration data for the system in Fig. 2 in a similar manner to structural model uncertainty (section 5.2.4):

1. Generation of the vibration data (section 3.1) from the system in Fig. 4 wherein the radial clearance of each SFD is corrupted with a prescribed level of radial clearance error (RCE). 
2. Solution of the inverse problem (section 3.2) using the original non-corrupted system (reference system) and the "real" vibration data obtained at step no.1.

3. Generation of the vibration data (section 3.1) from the "balanced" system in Fig. 8 with the SFDs corrupted with the prescribed RCEs used in step 1.

4. Comparison of the vibration data in step nos. 1 and 3 as a PVR (eq. (63)).

Figure 20 shows the PVRs obtained under conditions where both SFDs are simultaneously subjected to percentage RCEs of $-9,-7,-5,+5,+7,+9$, at $25 \mathrm{~dB}$ SNR, assuming no errors in the structural model (linear part). The balancing effectiveness is seen to be reasonably robust, with PVRs remaining above $50 \%$.
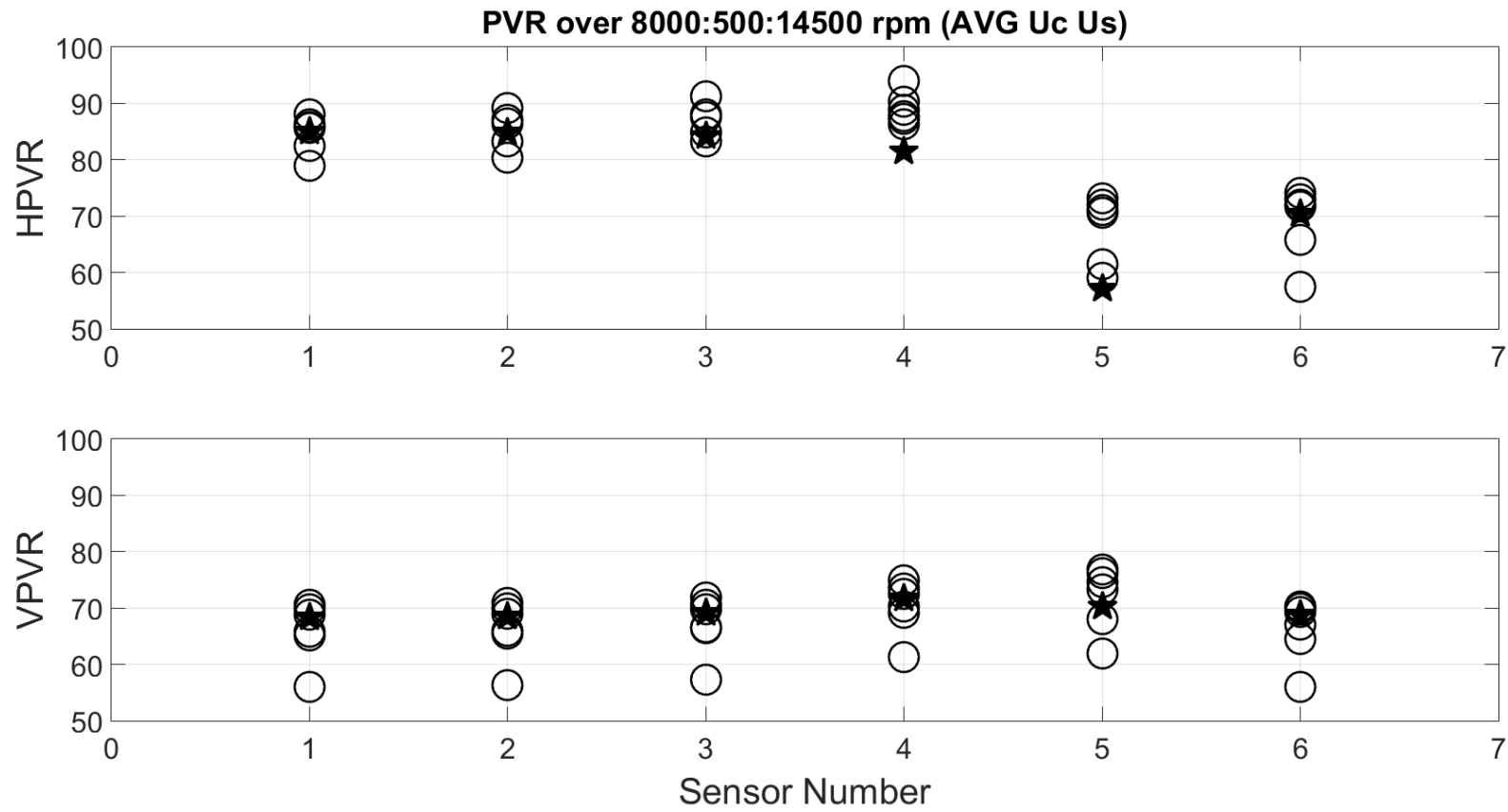

Fig. 20: Effect of SFD radial clearance uncertainty - PVRs when SFDs are simultaneously subjected to percentage radial clearance errors (RCEs) of $-9,-7,-5,+5,+7,+9$, in the presence of noise of $25 \mathrm{~dB}$ SNR, for $\gamma$ at $0^{\circ}$ (HPVR refers to $x$-direction, VPVR refers to $y$-direction)

$\star$ : no RCE; O : RCE )

\section{Conclusions}

This paper has introduced a non-invasive method for solving the quasi-implicit inverse problem for identifying the required balancing correction for a rotor with only one weak linear connection to the casing, apart from the nonlinear 
connections. This is typical of aero-engine designs that use a retainer spring with only one of the nonlinear squeezefilm damper (SFD) bearings that support the rotor within the casing. The badly conditioned explicit inverse problem relating the casing vibration readings to the equivalent unbalance in three prescribed planes was first set up using the RHBM method introduced in previous research for simpler configurations. An implicit problem relating the relative displacements at the SFD journals with the unbalance was then set up. An approximate method was devised to estimate the SFD journal displacements from the casing vibration using identified inverse SFD models based on Recurrent Neural Networks (RNNs). The data for training the RNNs of the inverse (force input/displacement output) models of both sprung and unsprung SFD bearings supporting the aero-engine HP rotor were generated using a recently introduced circular chirp excitation method. The information from the RNNs was used to partly solve the implicit inverse problem and combine it with the explicit inverse problem to enhance its condition. The methodology was validated using simulated casing vibration readings. The first part of the validation showed that the output from the RNNs correctly tracked the fundamental frequency of the true signals and the RNN-predicted orbits correlated reasonably well with the true ones. The correlation was better for the unsprung SFD RNN since its force input was more accurate due to the approximation made to estimate the SFD forces from the external vibration readings. The second part of the validation proved that the novel enhancement to the explicit inverse problem drastically improved its conditioning and was essential towards reducing the vibration of the system. The degree of repeatability of the method to changes in measurement phase datum was very satisfactory and comparable to that achieved for simpler configurations (with the unenhanced explicit method). The method was shown to achieve vibration reductions of at least $50 \%$ under combined conditions of $25 \mathrm{~dB}$ signal-to-noise ratio and $\pm 2 \%$ uncertainty in the structural model geometry / $\pm 9 \%$ uncertainty in the SFD radial clearance.

\section{Acknowledgements}

The authors would like to acknowledge the support from DGAPA-UNAM through its postdoctoral fellowship program named as "Programa de Becas Postdoctorales en la UNAM", likewise to the project PAPIIT IN117717 which is leaded by Meng. Alberto Reyes Solis. The authors are also grateful to The Higher Committee for Education Development in Iraq for financially supporting the current research 


\section{References}

[1] V. Dicken, P. Maaß, I. Menz, J. Niebsch, R. Ramlau, Nonlinear inverse unbalance reconstruction in rotor dynamics, Inverse Probl. Sci. Eng. 13 (2005) 507-543.

[2] S.G. Torres Cedillo, The identification of unbalance in a nonlinear squeeze-film damped system using an inverse method - a computational and experimental study, University of Manchester, 2015.

[3] S.G. Torres Cedillo, P. Bonello, An equivalent unbalance identification method for the balancing of nonlinear squeeze-film damped rotordynamic systems, J. Sound Vib. 360 (2016) 53-73.

[4] R.E.D. Bishop, G.M.L. Gladwell, The vibration and balancing of an unbalanced flexible rotor, J. Mech. Eng. Sci. 1 (1959) 66-77.

[5] T.P. Goodman, A least-squares method for computing balance corrections, J. Manuf. Sci. Eng. 86 (1964) 273277.

[6] M.S. Darlow, Balancing of high-speed machinery: Theory, methods and experimental results, Mech. Syst. Signal Process. 1 (1987) 105-134.

[7] W.C. Foiles, P.E. Allaire, E.J. Gunter, Review: Rotor Balancing, Shock Vib. 5 (1998).

[8] Y. Ishida, T. Yamamoto, Linear and nonlinear rotordynamics: a modern treatment with applications, John Wiley \& Sons, 2013.

[9] W.J. Chen, S.D. Rajan, H.D. Nelson, M. Rajan, Application of nonlinear programming for balancing rotor systems, Eng. Optim. 17 (1991) 79-90.

[10] J.M. Krodkiewski, J. Ding, N. Zhang, Identification of unbalance change using a non-linear mathematical model for multi-bearing rotor systems, J. Sound Vib. 169 (1994) 685-698.

[11] J.J. Ding, A. Al-Jumaily, A linear regression model for the identification of unbalance changes in rotating machines, J. Sound Vib. 231 (2000) 125-144.

[12] A.W. Lees, M.I. Friswell, The evaluation of rotor imbalance in flexibly mounted machines, J. Sound Vib. 208 (1997) 671-683.

[13] J.K. Sinha, A.W. Lees, M.I. Friswell, Estimating unbalance and misalignment of a flexible rotating machine from a single run-down, J. Sound Vib. 272 (2004) 967-989.

[14] A. W. Lees, J.K. Sinha, M.I. Friswell, The identification of the unbalance of a flexible rotating machine from a single rundown, J. Eng. Gas Turbines Power. 126 (2004) 416. 
[15] A.W. Lees, J.K. Sinha, M.I. Friswell, Model-based identification of rotating machines, Mech. Syst. Signal Process. 23 (2009) 1884-1893.

[16] R. Ramlau, V. Dicken, P. Maaß, C. Streller, A. Rienäcker, Inverse imbalance reconstruction in rotordynamics, ZAMM - J. Appl. Math. Mech. / Zeitschrift Für Angew. Math. Und Mech. 86 (2006) 385-399.

[17] S.G. Torres Cedillo, P. Bonello, Empirical identification of the inverse model of a squeeze-film damper bearing using neural networks and its application to a nonlinear inverse problem, J. Vib. Control. 24 (2016) $357-378$

[18] P. Bonello, P.M. Hai, Computational Studies of the Unbalance Response of a Whole Aero-Engine Model With Squeeze-Film Bearings, J. Eng. Gas Turbines Power. 132 (2009) 32504-32504-7.

[19] P. Bonello, M. J. Brennan and R. Holmes, Non-linear modelling of rotor dynamic systems with squeeze film dampers - an efficient integrated approach, J. Sound Vib. 249(4) (2002) 743-773.

[20] P. Bonello, P. Minh Hai, A receptance harmonic balance technique for the computation of the vibration of a whole aero-engine model with nonlinear bearings, J. Sound Vib. 324 (2009) 221-242.

[21] M.F. Bin Hassan, P. Bonello, A neural network identification technique for a foil-air bearing under variable speed conditions and its application to unbalance response analysis, J. Tribol. 139 (2016) 21501-21513.

[22] G. G. Al-Ghazal, P. Bonello, S.G. Torres Cedillo, Improved empirical identification of the inverse model of a squeeze-film damper bearing based on a recurrent neural network, in: Proc. ASME Turbo Expo (2018). paper no. GT2018-75777.

[23] M. Reymond, MSC . Nastran 2005, Matrix. 1 (2015).

[24] L. F. Shampine and M. W. Reichelt, The Matlab ODE suite. SIAM J. Sci. Comput. 18(1) (1997) 1-22.

[25] M.M. Dede, M. Dogan, R. Holmes, The damping capacity of a sealed squeeze film bearing, J. Tribol. 107 (1985) 411-418.

[26] D.J. Ewins, Modal testing: theory and practice, Research Studies Press, 2000.

[27] C.R. Vogel, Computational methods for inverse problems, Society for Industrial and Applied Mathematics, 2002.

[28] M.H. Beale, M.T. Hagan, H.B. Demuth, Neural Network Toolbox 7, User's Guide. MathWorks, 2010. 